STATE OF ALASKA

DEPARTMENT OF NATURAL RESOURCES

Alaska Geologic Materials Center Data Report No. 404

No. 404

Shennan I., Barlow N., Pierre K., Stuart-Taylor O., and Sea

Level Research Unit, Department of Geography, Durham

University, 2012, Diatom stratigraphy of Borehole TA8,

Portage, Alaska

Received July 2012

All data reports may be downloaded free of charge from the DGGS website. 


\section{Diatom stratigraphy of Borehole TA8, Portage, Alaska}

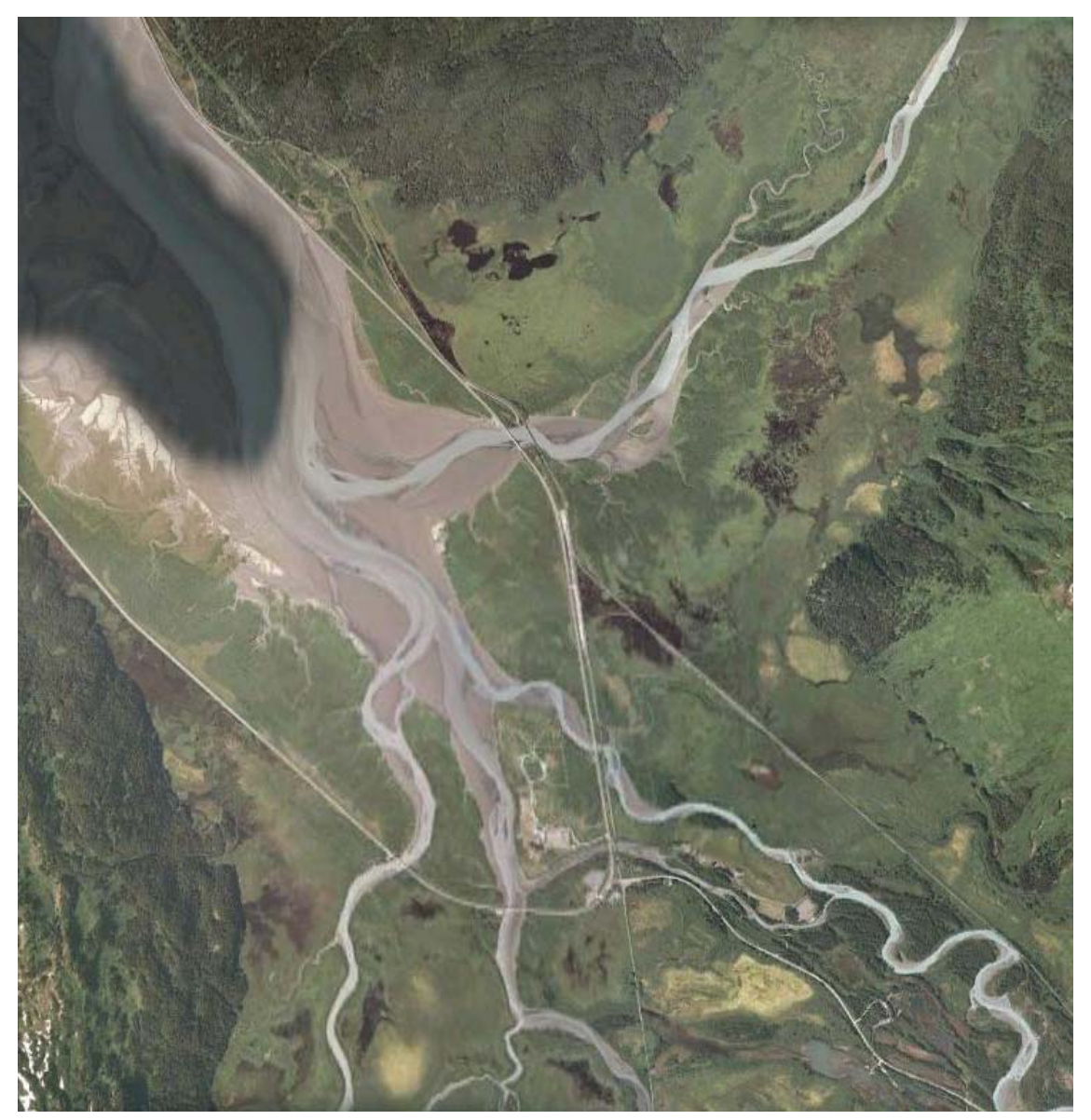

Ian Shennan, Natasha Barlow, Klara Pierre and Olivia Stuart-Taylor

Sea Level Research Unit

Department of Geography

Durham University

Science Laboratories

Durham DH1 3LE

UK

Corresponding author: ian.shennan@durham.ac.uk

July $31^{\text {st }}, 2012$ 


\section{Aim}

This report presents the results of diatom analyses of 69 samples taken from Borehole TA8 at Portage, Alaska. Borehole TA8 was drilled in 1988, with the lithology and radiocarbon ages reported shortly afterwards (Combellick, 1991). Following a preliminary study in 2011 that established good diatom preservation in various parts of the sequence, the archive cores were sampled at selected intervals to study the changes across major lithological boundaries as well as general trends through the whole sequence. We make some provisional comparisons with results from Girdwood and suggest options for possible further analysis.

\section{Methods}

In order to minimise disturbance of the archived cores we sampled across the organic layers radiocarbon dated by Combellick (1991) at 1 or $2 \mathrm{~cm}$ intervals, and at wider spaced intervals in the minerogenic layers (Appendix 1). Appendix 2 lists the diatom frequencies for each sample analysed.

\section{Results}

The following sections show a summary diatom diagram with brief commentary. We make a preliminary estimate of the change in elevation represented by the diatom and stratigraphic data and probable correlation with the sequence at Girdwood (Shennan et al., 2008). These are preliminary because: 1 ) in the absence of direct measurements of tide levels at Portage we use our measurements from Girdwood (Hamilton and Shennan, 2005) to calibrate the transfer function model estimates of elevation changes; 2) low water salinities at the head of Turnagain Arm may lead to poor matches between the fossil samples and our modern training set, Girdwood is the closest site for our modern samples; 3 ) the radiocarbon dates for the Portage core are based on bulk peat samples and may give ages that are too old (Hamilton et al., 2005); 4) a single borehole cannot provide evidence for the lateral continuity of a sharp stratigraphic contact, one of the key criteria suggested by (Nelson et al., 1996) so we require very strong support from the other evidence to make correlations with paleoseismic records at other sites. 


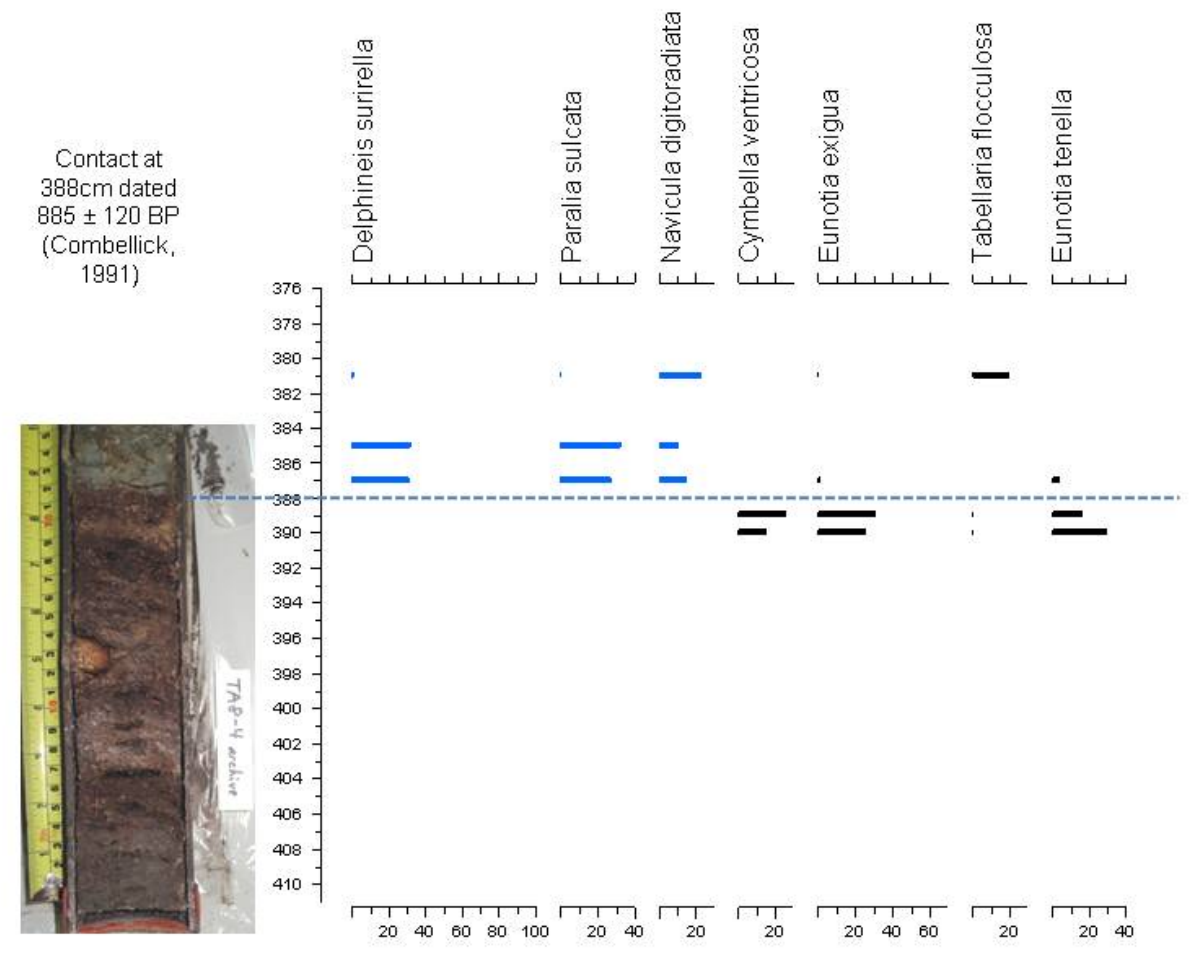

Figure 1: Summary diatom diagram of samples counted from core TA-8, across peat contact at $388 \mathrm{~cm}$ depth, showing only those species that reached $>10 \%$. Blue shows species that favour marine and brackish water. See Appendix 2 for full data. Equivalent layer dated by Combellick (1991).

The sharp peat-silt contact and clear change in diatom assemblages indicate a rapid change in environment, with subsidence estimated at $1.6 \pm 0.5 \mathrm{~m}(1 \sigma)$ using our transfer function model. This is very similar to AD 1964 and the estimate for the penultimate great earthquake at Girdwood (Shennan and Hamilton, 2006). 


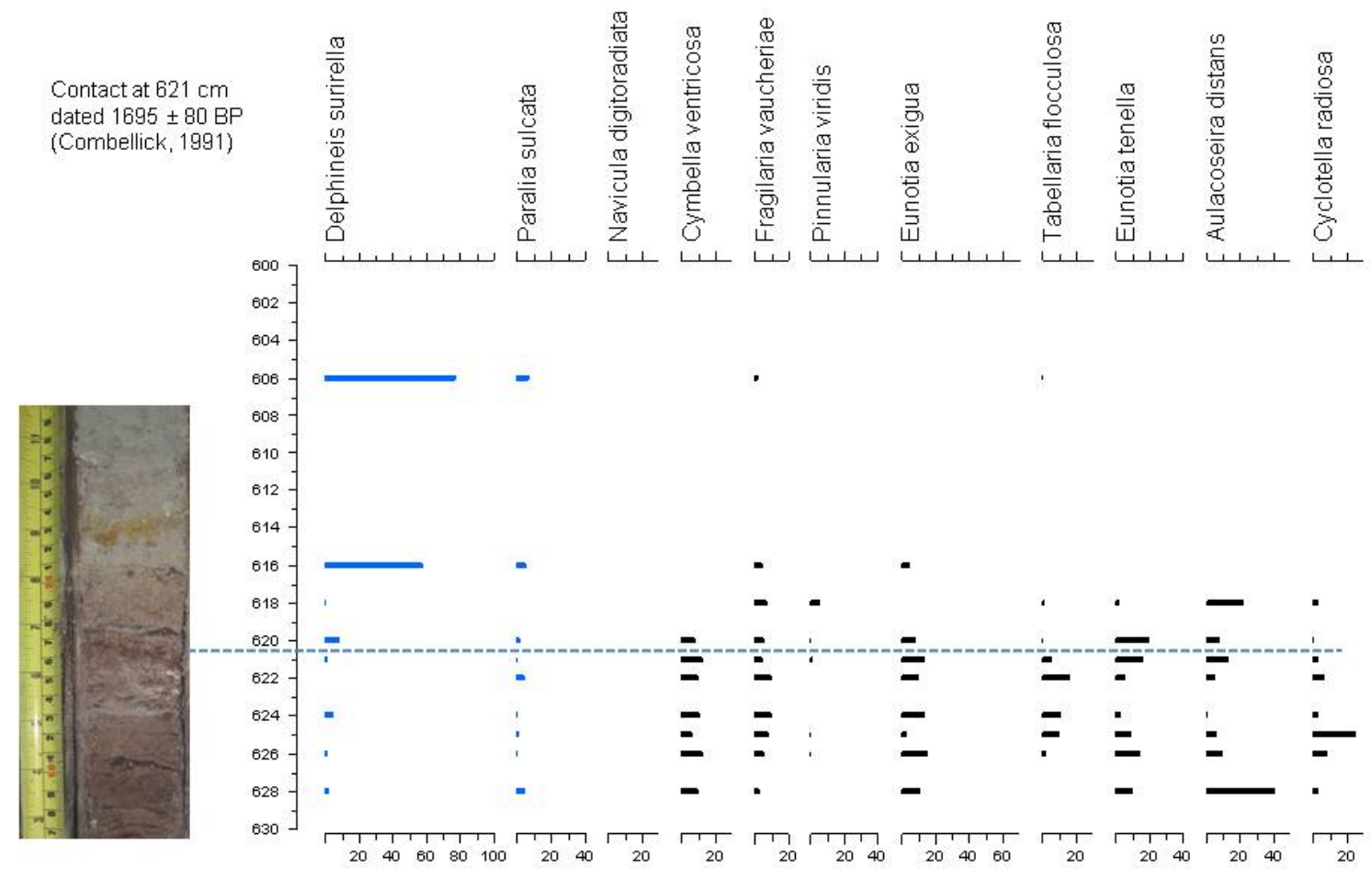

Figure 2: Summary diatom diagram of samples counted from core TA-8, across peat contact at $621 \mathrm{~cm}$ depth, showing only those species that reached $>10 \%$. Blue shows species that favour marine and brackish water. See Appendix 2 for full data. Equivalent layer dated by Combellick (1991).

Visual inspection of the stratigraphy suggests likely sediment mixing at the peat-silt boundary. The transfer function model estimates indicate subsidence in the order of $1.2 \pm 0.5 \mathrm{~m}$. This compares with $1.4 \pm 0.3 \mathrm{~m}$ $\sim 1500$ BP at Girdwood. 


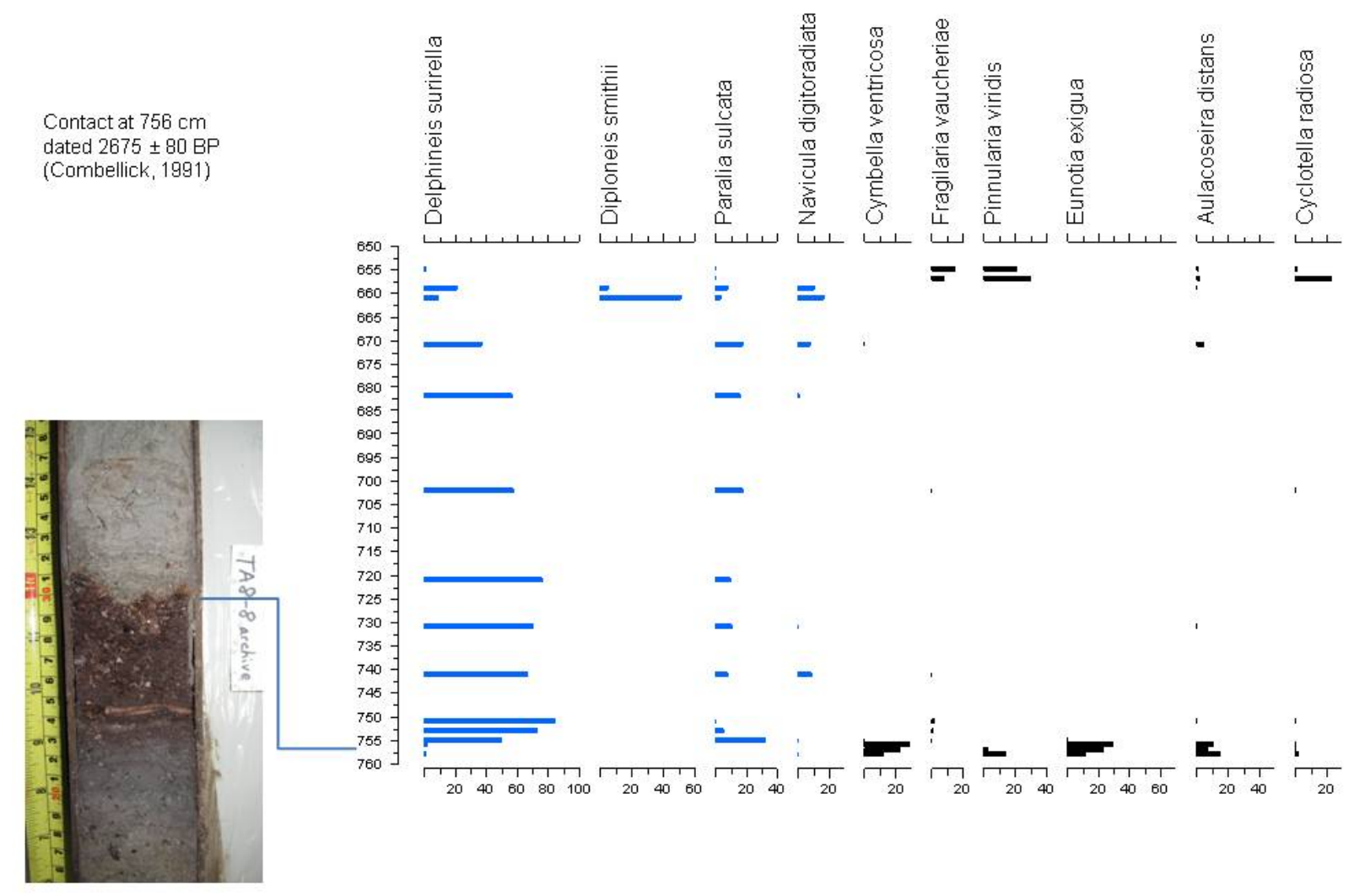

Figure 3: Summary diatom diagram of samples counted from core TA-8, across peat contact at $756 \mathrm{~cm}$ depth, showing only those species that reached $>10 \%$. Blue shows species that favour marine and brackish water. See Appendix 2 for full data. Equivalent layer dated by Combellick (1991). Silt layer, more laminated below $750 \mathrm{~cm}$, continues to gradual boundary to peat at top of diagram (base of the same peat layer in figure 2).

The wavy boundary at the top of the peat layer suggests possible erosion of the contact. The change from predominantly freshwater diatom species in the peat to marine and brackish species in the silt unit suggests rapid submergence at $756 \mathrm{~cm}$, and a gradual return to peat accumulation above $660 \mathrm{~cm}$. The transfer function model estimates $1.4 \pm 0.5 \mathrm{~m}$ subsidence across the contact at $756 \mathrm{~cm}$. Given the possibility of erosion at the contact and the limitations of the radiocarbon dating we cannot be certain of the correlation with Girdwood, but given the sequences above and below this peat, it appears likely that this peat records the same earthquake dated $\sim 2100 \mathrm{BP}$ at Girdwood, with $1.2 \pm 0.3 \mathrm{~m}$ subsidence. 


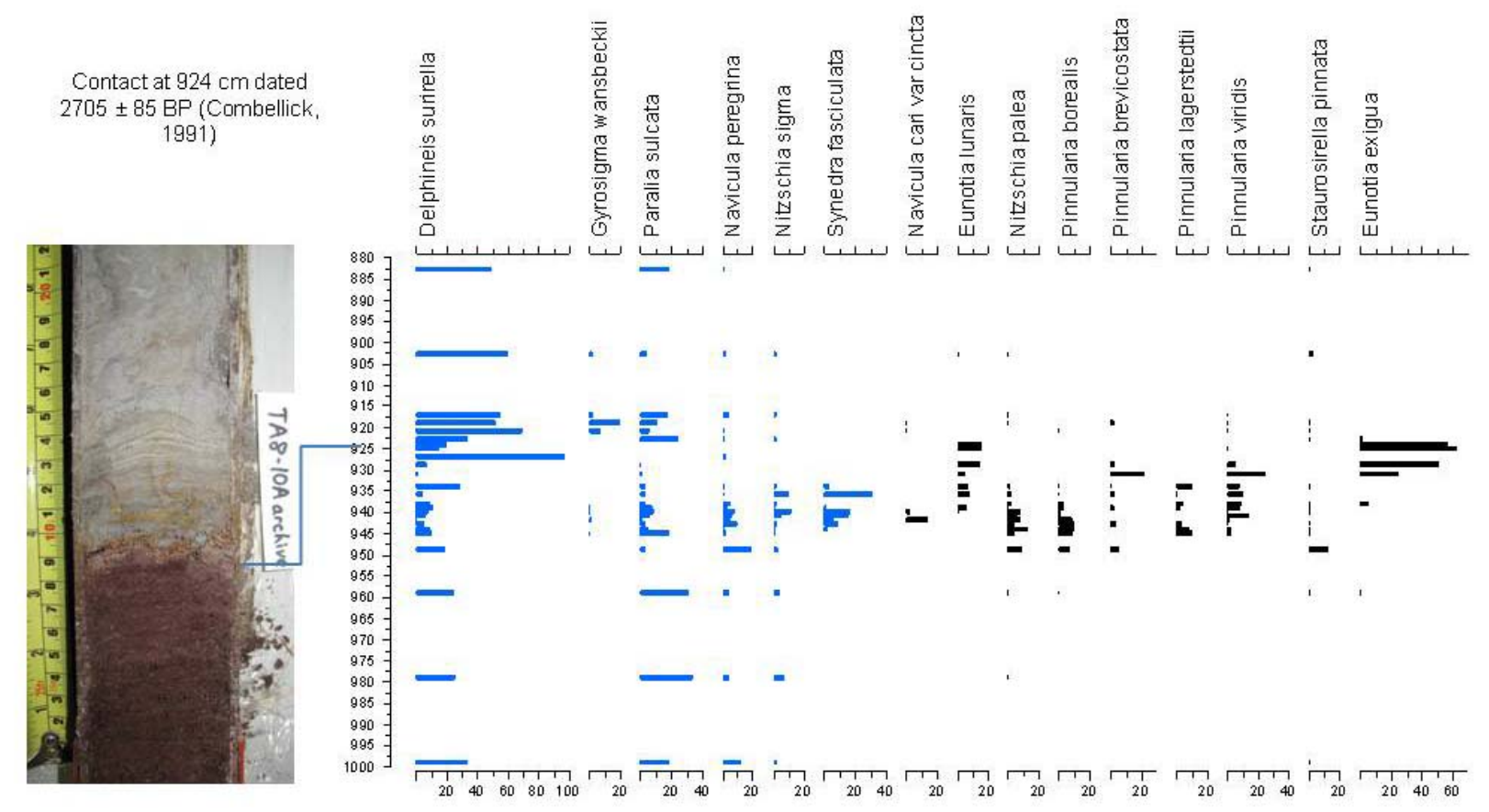

Figure 4: Summary diatom diagram of samples counted from core TA-8, across peat contact at $924 \mathrm{~cm}$ depth, showing only those species that reached $>10 \%$. Blue shows species that favour marine and brackish water. See Appendix 2 for full data. Equivalent layer dated by Combellick (1991).

\begin{tabular}{|l|l|}
\hline Depth cm & Composition \\
\hline 883 & silt/fine sand \\
\hline 903 & silt/fine sand \\
\hline 917 & fine sand \\
\hline 919 & laminated silt \\
\hline 921 & laminated silt \\
\hline 923 & silt \\
\hline 924 & peat \\
\hline 925 & oeat \\
\hline 927 & peat \\
\hline 929 & peat \\
\hline 931 & peat \\
\hline 934 & peat \\
\hline 936 & peat \\
\hline 938 & peaty-silt transition \\
\hline 939 & silt \\
\hline 940 & silt \\
\hline 941 & silt \\
\hline 942 & peaty clay \\
\hline 943 & peaty clay \\
\hline 944 & peaty clay \\
\hline 945 & slightly peaty clay \\
\hline 949 & slightly peaty clay \\
\hline 959 & clay silt \\
\hline 979 & laminated silt \\
\hline 999 & laminated silt \\
\hline & \\
\hline
\end{tabular}

Table 1. Copy of sampling notes recoding the $0.5 \mathrm{cc}$ sediment sample taken at each level, 883 to $999 \mathrm{~cm}$ (full list in Appendix 1).

The diatom assemblages show a rapid change from predominantly freshwater species to marine and brackish species across the top contact of the peat layer, at $924 \mathrm{~cm}$. Poor diatom preservation at 927 $\mathrm{cm}$ allowed gave a count of only 37 diatoms, so the peak in Delphineis surirella at this level is not reliable. The transfer function model estimates subsidence of $1.3 \pm 0.5 \mathrm{~m}$. Provisional correlation with Girdwood is the earthquake $1.4 \pm 0.3 \mathrm{~m}, \sim 2500 \mathrm{BP}$. The peaty clay silt - peat sequence, 945 to $938 \mathrm{~cm}$, coincides with temporary peaks of Nitzschia sigma and Synedra fasciculata, and the transfer function predicts possible submergence in the order of $0.3 \pm 0.6 \mathrm{~m}$. This may be the equivalent of a peat-silt couplet at Girdwood, 2800 BP deemed non-seismic by Shennan and Hamilton (2006). 


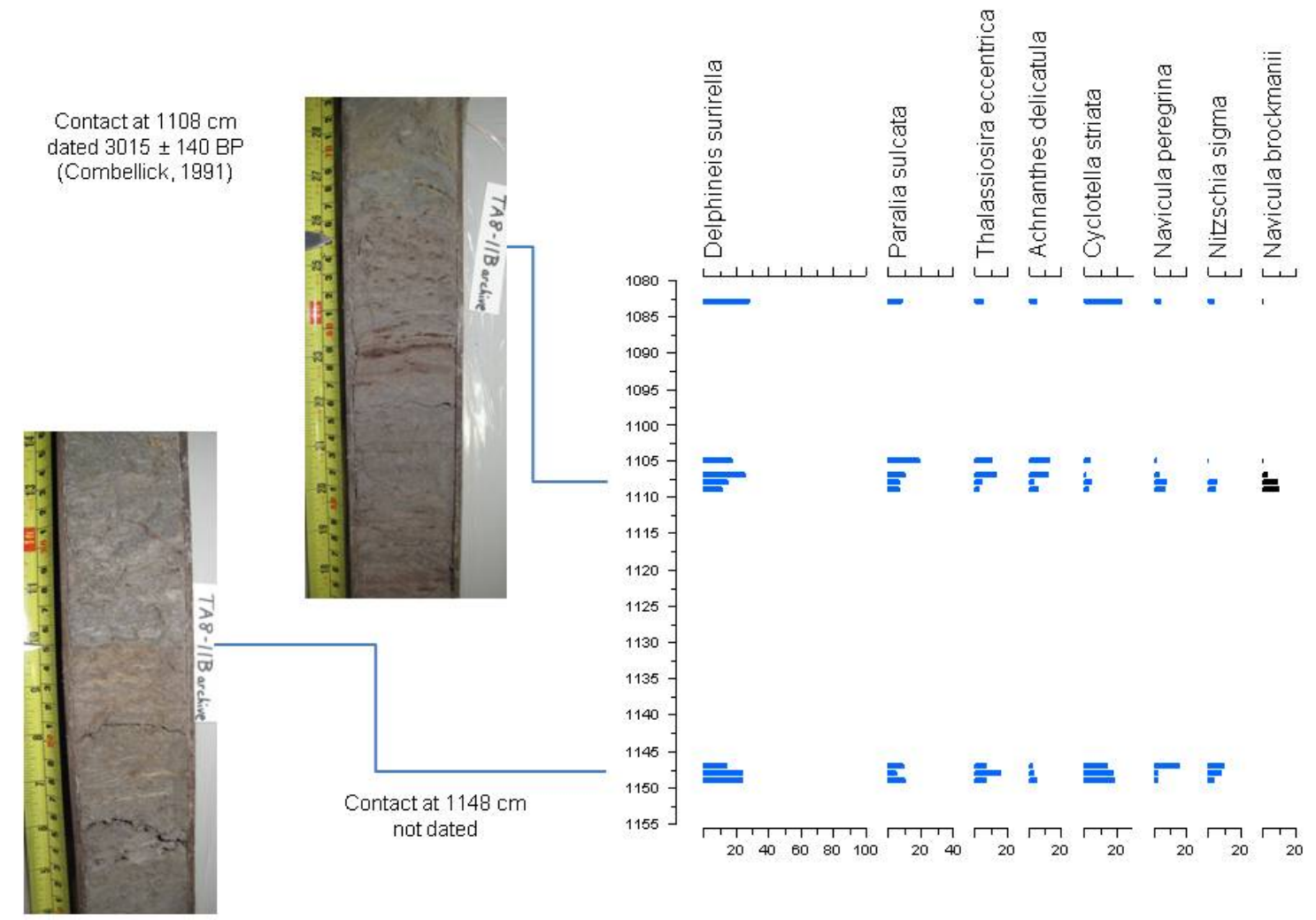

Figure 5: Summary diatom diagram of samples counted from core TA-8, across contacts at 1108 and $1148 \mathrm{~cm}$ depth, showing only those species that reached $>10 \%$. Blue shows species that favour marine and brackish water. See Appendix 2 for full data. Equivalent layer dated by Combellick (1991).

Changes in organic content suggest two possible rapid submergence events, at $1108 \mathrm{~cm}$ and $1148 \mathrm{~cm}$. Both record changes in diatom assemblages, in mainly marine and brackish species. The transfer function model suggests possible submergence, $0.6 \pm 0.6 \mathrm{~m}$ at $1108 \mathrm{~cm}$, but no predicted submergence, $-0.2 \pm 0.6 \mathrm{~m}$. 

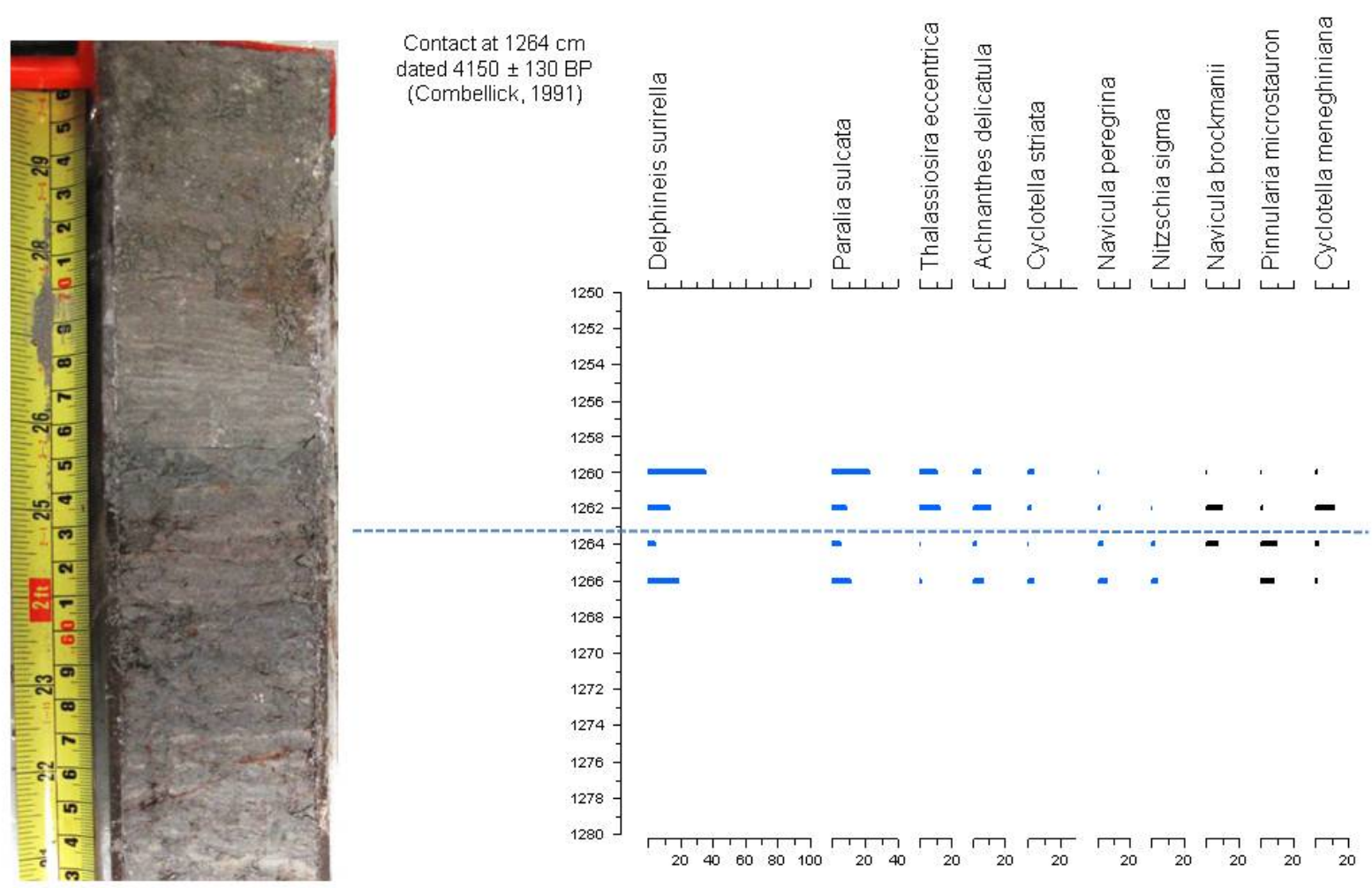

Figure 6: Summary diatom diagram of samples counted from core TA-8, across contacts at $1264 \mathrm{~cm}$ depth, showing only those species that reached $>10 \%$. Blue shows species that favour marine and brackish water. See Appendix 2 for full data. Equivalent layer dated by Combellick (1991).

A change in organic content $1264 \mathrm{~cm}$ suggests possible submergence. Changes in diatom frequencies possibly suggest gradual trends rather than an abrupt change but we would require further counts to confirm this. The transfer function model predicts submergence of $0.6 \pm 0.6 \mathrm{~m}$. 


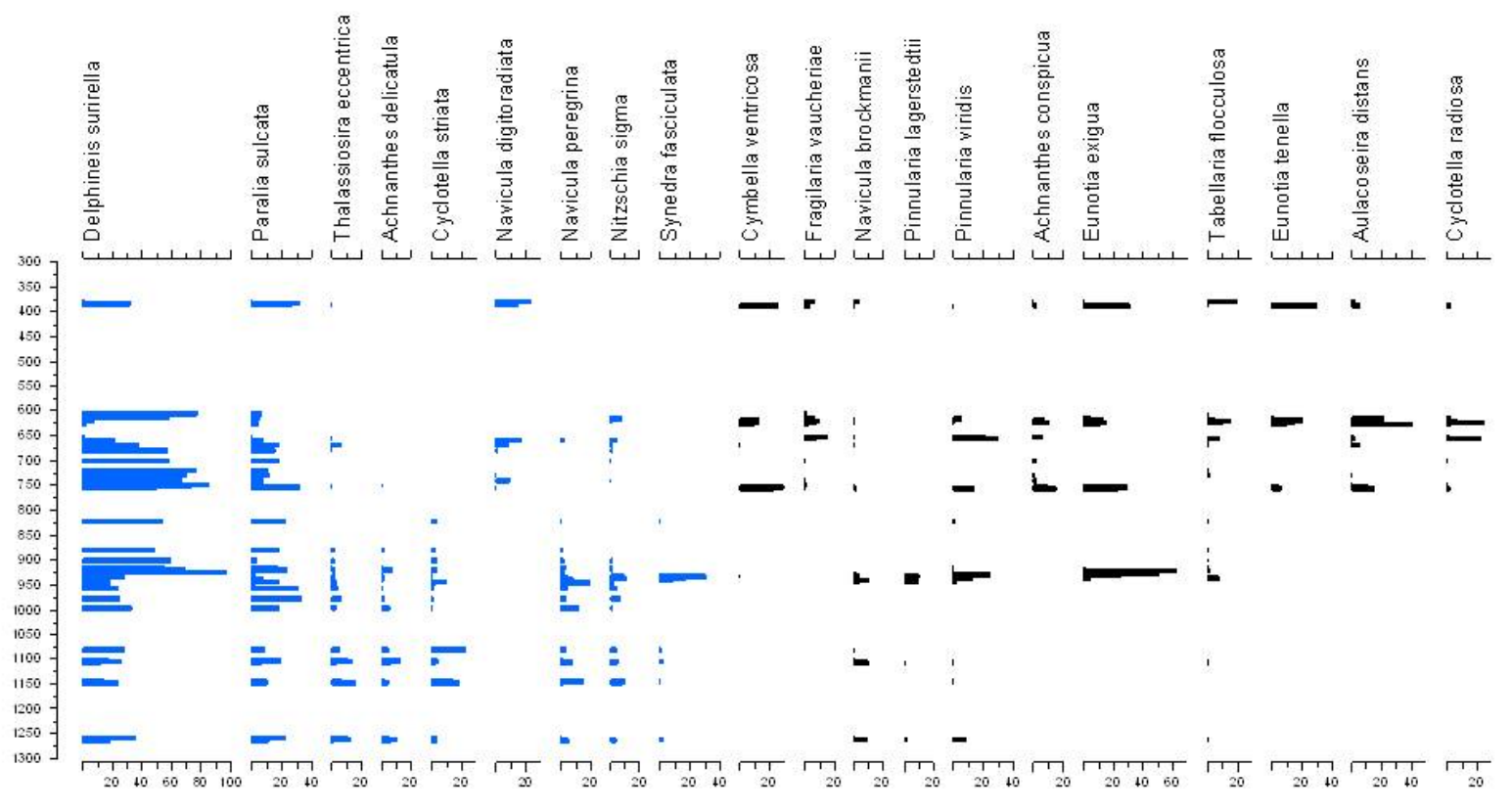

Figure 7: Summary diatom diagram of samples counted from core TA-8, from 300 to $1300 \mathrm{~cm}$ depth, showing only those species that reached $>20 \%$ in more than one level. Blue shows species that favour marine and brackish water. See Appendix 2 for full data.

Figure 7: Summary diatom diagram of samples counted from core TA-8, from 300 to $1300 \mathrm{~cm}$ depth, showing only those species that reached $>20 \%$ in more than one level. Blue shows species that favour marine and brackish water. See Appendix 2 for full data.

The diatom assemblage data down core to $1300 \mathrm{~cm}$ depth confirm sediment accumulation in a tidal environment, with predominantly intertidal minerogenic and organic marsh sediments. Both the sediment stratigraphy and diatom assemblages reflect the spatial changes of an infilling embayment superimposed on generally rising relative sea level and multiple earthquake deformation cycles. No well developed peat layers occur below $950 \mathrm{~cm}$ core depth and any evidence of coseismic submergence occurs within probable low marsh or tidal flat paleoenvironments. These give less precise reconstructions of elevation changes. We would require a borehole further inland to record the peat-forming marsh communities of that period. Above $950 \mathrm{~cm}$ core depth, diatom assemblages show how peat layers developed in freshwater conditions, though still controlled by tide levels. Only two samples, 388 and $390 \mathrm{~cm}$, show no clearly discernible marine or brackish influence. 


\section{Conclusions}

Fossil diatom abundances provide a record of late of sediment infilling at the head of Turnagain Arm, revealing $\sim 13 \mathrm{~m}$ net relative sea level rise superimposed with elevation changes associated with multiple earthquake deformation cycles. Preliminary correlation with Girdwood indicates four prehistoric earthquakes with similar coseismic subsidence. Events older than $~ 3000$ BP are less precisely recorded as the Portage location was in a more open tidal flat environment at that time.

Analysis of further samples, both those already taken from the cores and further samples from the cores in store, would provide more detailed analysis of the paleoenvironmental changes at the head of Turnagain Arm. They would provide information for numerous lines of inquiry, not just paleoseismology, but other fields, including sediment dynamics, sediment compaction, glacial isostatic adjustment, sediment delivery during the Little Ice Age and crustal loading.

The key to virtually all of these possible developments is an improved chronology. This requires sampling of the core and careful picking of plant macrofossils, or other suitable material, for high precision/small sample radiocarbon dating. These are methods were not available when the borehole was drilled. The archive core has been well preserved and should provide multiple samples suitable for dating. 


\section{References (including those cited in Appendices)}

Combellick, R.A., 1991. Palaeoseismicity of the Cook Inlet region, Alaska: Evidence from peat stratigraphy in Turnagain and Knik Arms. Alaska Division of Geological and Geophysical Surveys Professional Report, 112: 1-52.

Hamilton, S. and Shennan, I., 2005. Late Holocene relative sea-level changes and the earthquake deformation cycle around upper Cook Inlet, Alaska. Quaternary Science Reviews, 24(12-13): 14791498.

Hamilton, S., Shennan, I., Combellick, R., Mulholland, J. and Noble, C., 2005. Evidence for two great earthquakes at Anchorage, Alaska and implications for multiple great earthquakes through the Holocene. Quaternary Science Reviews, 24(18-19): 2050-2068.

Hartley, B., Barber, H.G. and Carter, J.R., 1996. An atlas of British diatoms. Biopress, Bristol, 601 pp.

Hemphill-Haley, E., 1993. Taxonomy of recent and fossil (Holocene) diatoms (Bacillariophyta) from northern Willapa Bay, Washington. U.S. Geological Survey Open File Report, 93-289: 1-151.

Nelson, A.R., Shennan, I. and Long, A.J., 1996. Identifying coseismic subsidence in tidal-wetland stratigraphic sequences at the Cascadia subduction zone of western North America. Journal of Geophysical Research, 101: 6115-6135.

Palmer, A.J.M. and Abbott, W.H., 1986. Diatoms as indicators of sea-level change. In: O. Van de Plassche (Editor), Sea-level research: a manual for the collection and evaluation of data. Geo Books, Norwich, pp. 457-488.

Patrick, R. and Reimer, C.W., 1966. The Diatoms of the United States. Volume 1. Monographs of The Academy of Natural Sciences of Philadelphia No 13. Academy of Natural Sciences of Philadelphia, Philadelphia, $688 \mathrm{pp}$.

Patrick, R. and Reimer, C.W., 1975. The Diatoms of the United States. Volume 2, Part 1. Monographs of The Academy of Natural Sciences of Philadelphia No 13. The Academy of Natural Sciences of Philadelphia, Philadelphia, 213 pp.

Shennan, I., Barlow, N. and Combellick, R., 2008. Palaeoseismological records of multiple great earthquakes in south-central Alaska - a 4000 year record at Girdwood. In: J.T. Freymueller, P.J. Haeussler, R. Wesson and G. Ekstrom (Editors), Active tectonics and seismic potential of Alaska. Geophysical Monograph Series, Volume 179. American Geophysical Union Washington, pp. 185-199.

Shennan, I. and Hamilton, S., 2006. Coseismic and pre-seismic subsidence associated with great earthquakes in Alaska. Quaternary Science Reviews, 25(1-2): 1-8.

Van der Werff, A. and Huls, H., 1958-1974. Diatomeeenflora van Nederland. 8 parts. Published privately, De Hoef, The Netherlands. 


\section{Appendix 1}

Samples taken from the archive cores, August 2011. Samples measured from the base of the storage tube for each core section. Some cores showed slight shrinkage or settlement during storage so boundaries of units may differ slightly from those reported by Combellick (1991) and the preliminary report (2011). Depth from surface calculated from the base of core sections data reported by Combellick (1991).

\begin{tabular}{|c|c|c|c|c|c|}
\hline $\begin{array}{l}\text { Archive } \\
\text { core label }\end{array}$ & $\begin{array}{l}\text { sample } \\
\#\end{array}$ & $\begin{array}{l}\text { Measured } \\
\text { distance } \\
\text { above } \\
\text { base of } \\
\text { tube } \\
\mathrm{cm}\end{array}$ & $\begin{array}{l}\text { Depth } \\
\text { from } \\
\text { surface } \\
\mathrm{cm}\end{array}$ & Composition & $\begin{array}{c}\text { Equivalent } 14 C \text { sample } \\
\text { by Combellick (1991) }\end{array}$ \\
\hline TA8-4 & 18 & 51 & 357 & silt & \\
\hline TA8-4 & 17 & 39 & 369 & silt & \\
\hline TA8-4 & 19 & 33 & 375 & silt + organics & \\
\hline TA8-4 & 16 & 31 & 377 & silt & \\
\hline TA8-4 & 15 & 27 & 381 & silt & \\
\hline TA8-4 & 14 & 23 & 385 & silt & \\
\hline TA8-4 & 13 & 21 & 387 & base of silt & \\
\hline TA8-4 & 12 & 20 & 388 & top of peat & GX-15218: 885+-120 BP \\
\hline TA8-4 & 11 & 19 & 389 & peat & \\
\hline TA8-4 & 10 & 18 & 390 & peat & \\
\hline TA8-4 & 9 & 16 & 392 & peat & \\
\hline TA8-4 & 8 & 13 & 395 & peat & \\
\hline TA8-4 & 7 & 10 & 398 & peat & \\
\hline TA8-4 & 6 & 9 & 399 & peaty silt & \\
\hline TA8-4 & 5 & 8 & 400 & peaty silt & \\
\hline TA8-4 & 4 & 7 & 401 & peat below silt & \\
\hline TA8-4 & 3 & 5 & 403 & base of peat & \\
\hline TA8-4 & 2 & 3 & 405 & silt & \\
\hline TA8-4 & 1 & 1 & 407 & silt & \\
\hline TA8-5A & 22 & 40 & 423 & silt & \\
\hline TA8-5A & 21 & 25 & 438 & silt & \\
\hline TA8-5A & 20 & 5 & 458 & silt & \\
\hline TA8-5B & 25 & 45 & 488 & silt & \\
\hline TA8-5B & 24 & 25 & 508 & silt & \\
\hline TA8-5B & 23 & 5 & 528 & silt & \\
\hline TA8-6 & 40 & 61 & 576 & silt & \\
\hline TA8-6 & 39 & 51 & 586 & silt & \\
\hline TA8-6 & 38 & 41 & 596 & silt & \\
\hline TA8-6 & 37 & 31 & 606 & silt & \\
\hline TA8-6 & 36 & 21 & 616 & silt & \\
\hline TA8-6 & 35 & 19 & 618 & silt \& plant fragments & \\
\hline TA8-6 & 34 & 17 & 620 & silt & \\
\hline TA8-6 & 33 & 16 & 621 & peat & GX-15219: $1695+-80 \mathrm{BP}$ \\
\hline TA8-6 & 32 & 15 & 622 & organic silt & \\
\hline TA8-6 & 31 & 13 & 624 & silt & \\
\hline TA8-6 & 30 & 12 & 625 & peat \& silt & \\
\hline TA8-6 & 29 & 11 & 626 & peat \& silt & \\
\hline
\end{tabular}




\begin{tabular}{|c|c|c|c|c|c|}
\hline $\begin{array}{l}\text { Archive } \\
\text { core label }\end{array}$ & $\begin{array}{c}\text { sample } \\
\#\end{array}$ & $\begin{array}{l}\text { Measured } \\
\text { distance } \\
\text { above } \\
\text { base of } \\
\text { tube } \\
\mathrm{cm}\end{array}$ & $\begin{array}{l}\text { Depth } \\
\text { from } \\
\text { surface } \\
\mathrm{cm}\end{array}$ & Composition & $\begin{array}{c}\text { Equivalent } 14 \text { c sample } \\
\text { by Combellick (1991) }\end{array}$ \\
\hline TA8-6 & 28 & 9 & 628 & peat & \\
\hline TA8-6 & 27 & 6 & 631 & peat & \\
\hline TA8-6 & 26 & 2 & 635 & peat & \\
\hline TA8-7 & 48 & 57 & 653 & peat (below silt contamination) & \\
\hline TA8-7 & 47 & 55 & 655 & peat & \\
\hline TA8-7 & 46 & 53 & 657 & clay peat & \\
\hline TA8-7 & 45 & 51 & 659 & organic clay & \\
\hline TA8-7 & 44 & 49 & 661 & silt \& clay & \\
\hline TA8-7 & 43 & 39 & 671 & silt & \\
\hline TA8-7 & 42 & 28 & 682 & silt & \\
\hline TA8-7 & 41 & 8 & 702 & silt & \\
\hline TA8-8 & 63 & 64 & 721 & silt & \\
\hline TA8-8 & 62 & 54 & 731 & silt & \\
\hline TA8-8 & 61 & 44 & 741 & silt & \\
\hline TA8-8 & 60 & 34 & 751 & laminated silt & \\
\hline TA8-8 & 59 & 32 & 753 & laminated silt & \\
\hline TA8-8 & 58 & 30 & 755 & laminated silt & \\
\hline TA8-8 & 57 & 29 & 756 & top of peat & GX-15220: $2675+-80$ BP \\
\hline TA8-8 & 56 & 28 & 757 & peat & \\
\hline TA8-8 & 55 & 27 & 758 & peat & \\
\hline TA8-8 & 54 & 26 & 759 & peat & \\
\hline TA8-8 & 53 & 25 & 760 & peat & \\
\hline TA8-8 & 52 & 23 & 762 & organic clay & \\
\hline TA8-8 & 51 & 21 & 764 & clay peat & \\
\hline TA8-8 & 50 & 13 & 772 & laminated silt & \\
\hline TA8-8 & 49 & 3 & 782 & laminated silt & \\
\hline TA8-9 & 65 & 40 & 823 & laminated silt & \\
\hline TA8-9 & 64 & 10 & 853 & laminated silt & \\
\hline TA8-10A & 76 & 50 & 883 & silt/fine sand & \\
\hline TA8-10A & 75 & 30 & 903 & silt/fine sand & \\
\hline TA8-10A & 74 & 16 & 917 & fine sand & \\
\hline TA8-10A & 73 & 14 & 919 & laminated silt & \\
\hline TA8-10A & 72 & 12 & 921 & laminated silt & \\
\hline TA8-10A & 71 & 10 & 923 & silt & \\
\hline TA8-10A & 70 & 9 & 924 & peat & GX-15221: 2705+-85 BP \\
\hline TA8-10A & 69 & 8 & 925 & peat & \\
\hline TA8-10A & 68 & 6 & 927 & peat & \\
\hline TA8-10A & 67 & 4 & 929 & peat & \\
\hline TA8-10A & 66 & 2 & 931 & peat & \\
\hline TA8-10B & 90 & 75 & 934 & peat & \\
\hline TA8-10B & 89 & 73 & 936 & peat & \\
\hline TA8-10B & 88 & 71 & 938 & peaty-silt transition & \\
\hline TA8-10B & 87 & 70 & 939 & silt & \\
\hline TA8-10B & 86 & 69 & 940 & silt & \\
\hline
\end{tabular}




\begin{tabular}{|c|c|c|c|c|c|}
\hline $\begin{array}{l}\text { Archive } \\
\text { core label }\end{array}$ & $\begin{array}{c}\text { sample } \\
\#\end{array}$ & $\begin{array}{l}\text { Measured } \\
\text { distance } \\
\text { above } \\
\text { base of } \\
\text { tube } \\
\mathrm{cm}\end{array}$ & $\begin{array}{l}\text { Depth } \\
\text { from } \\
\text { surface } \\
\mathrm{cm}\end{array}$ & Composition & $\begin{array}{l}\text { Equivalent } 14 \text { C sample } \\
\text { by Combellick (1991) }\end{array}$ \\
\hline TA8-10B & 85 & 68 & 941 & silt & \\
\hline TA8-10B & 84 & 67 & 942 & peaty clay & \\
\hline TA8-10B & 83 & 66 & 943 & peaty clay & \\
\hline TA8-10B & 82 & 65 & 944 & peaty clay & \\
\hline TA8-10B & 81 & 64 & 945 & slightly peaty clay & \\
\hline TA8-10B & 80 & 60 & 949 & slightly peaty clay & \\
\hline TA8-10B & 79 & 50 & 959 & clay silt & \\
\hline TA8-10B & 78 & 30 & 979 & laminated silt & \\
\hline TA8-10B & 77 & 10 & 999 & laminated silt & \\
\hline TA8-11A & 93 & 50 & 1037 & silt & \\
\hline TA8-11A & 92 & 28 & 1059 & silt & \\
\hline TA8-11A & 91 & 4 & 1083 & silt & \\
\hline TA8-11B & 116 & 68 & 1105 & silt & \\
\hline TA8-11B & 115 & 66 & 1107 & silt & \\
\hline TA8-11B & 114 & 65 & 1108 & $\begin{array}{r}\text { top contact of organic laminated } \\
\text { silt }\end{array}$ & GX-15222: 3015+-140 BP \\
\hline TA8-11B & 113 & 64 & 1109 & organic laminated silt & \\
\hline TA8-11B & 112 & 63 & 1110 & organic laminated silt & \\
\hline TA8-11B & 111 & 62 & 1111 & organic laminated silt & \\
\hline TA8-11B & 110 & 60 & 1113 & organic laminated silt & \\
\hline TA8-11B & 109 & 58 & 1115 & organic laminated silt & \\
\hline TA8-11B & 108 & 54 & 1119 & organic laminated silt & \\
\hline TA8-11B & 107 & 48 & 1125 & organic laminated silt & \\
\hline TA8-11B & 106 & 42 & 1131 & organic laminated silt & \\
\hline TA8-11B & 105 & 36 & 1137 & laminated silt + plant fragments & \\
\hline TA8-11B & 104 & 28 & 1145 & laminated silt + plant fragments & \\
\hline TA8-11B & 103 & 26 & 1147 & laminated silt & \\
\hline TA8-11B & 102 & 25 & 1148 & $\begin{array}{r}\text { top contact of organic laminated } \\
\text { silt }\end{array}$ & \\
\hline TA8-11B & 101 & 24 & 1149 & organic laminated silt & \\
\hline TA8-11B & 100 & 23 & 1150 & organic laminated silt & \\
\hline TA8-11B & 99 & 21 & 1152 & organic laminated silt & \\
\hline TA8-11B & 98 & 19 & 1154 & organic laminated silt & \\
\hline TA8-11B & 97 & 17 & 1156 & laminated silt + plant fragments & \\
\hline TA8-11B & 96 & 13 & 1160 & laminated silt + plant fragments & \\
\hline TA8-11B & 95 & 9 & 1164 & laminated silt & \\
\hline TA8-11B & 94 & 5 & 1168 & laminated silt & \\
\hline TA8-12A & 118 & 45 & 1205 & laminated silt & \\
\hline TA8-12A & 117 & 14 & 1236 & laminated silt & \\
\hline TA8-12B & 126 & 68 & 1258 & laminated silt & \\
\hline TA8-12B & 125 & 66 & 1260 & laminated silt & \\
\hline TA8-12B & 124 & 64 & 1262 & laminated silt & \\
\hline TA8-12B & 123 & 62 & 1264 & laminated silt + plant fragments & GX-15223: 4150+-130 BP \\
\hline TA8-12B & 122 & 60 & 1266 & laminated silt + plant fragments & \\
\hline
\end{tabular}




\begin{tabular}{|c|c|c|c|c|c|}
\hline $\begin{array}{l}\text { Archive } \\
\text { core label }\end{array}$ & $\begin{array}{c}\text { sample } \\
\#\end{array}$ & $\begin{array}{l}\text { Measured } \\
\text { distance } \\
\text { above } \\
\text { base of } \\
\text { tube } \\
\mathrm{cm}\end{array}$ & $\begin{array}{l}\text { Depth } \\
\text { from } \\
\text { surface } \\
\mathrm{cm}\end{array}$ & Composition & $\begin{array}{l}\text { Equivalent } 14 \text { C sample } \\
\text { by Combellick (1991) }\end{array}$ \\
\hline TA8-12B & 121 & 50 & 1276 & laminated silt + plant fragments & \\
\hline TA8-12B & 120 & 30 & 1296 & laminated silt + plant fragments & \\
\hline TA8-12B & 119 & 12 & 1314 & laminated silt + plant fragments & \\
\hline TA8-13A & 129 & 30 & 1372 & dark grey lamination & \\
\hline TA8-13A & 128 & 29 & 1373 & light grey lamination & \\
\hline TA8-13A & 127 & 14 & 1388 & laminated silt & \\
\hline TA8-13B & 131 & 48 & 1430 & laminated silt & \\
\hline TA8-13B & 130 & 34 & 1444 & unlaminated silt & \\
\hline TA8-14A & 134 & 15 & 1538 & laminated silt & \\
\hline TA8-14A & 133 & 3 & 1550 & silt, possible organic matter & \\
\hline TA8-14A & 132 & 2 & 1551 & silt & \\
\hline TA8-14B & 140 & 54 & 1574 & laminated silt & \\
\hline TA8-14B & 139 & 44 & 1584 & laminated silt, possibly tephra & \\
\hline TA8-14B & 138 & 32 & 1596 & laminated silt & \\
\hline TA8-14B & 137 & 22 & 1606 & laminated silt & \\
\hline TA8-14B & 136 & 18 & 1610 & laminated silt, possibly tephra & \\
\hline TA8-14B & 135 & 6 & 1622 & laminated silt & \\
\hline
\end{tabular}




\section{Appendix 2}

\section{Diatom data}

Preparation of diatom samples followed standard laboratory methods (Palmer and Abbott, 1986). The two key reference volumes for coastal and estuarine diatoms are based on NW European material (Hartley et al., 1996; Van der Werff and Huls, 1958-1974) together with supplementary information from the Pacific Northwest (Hemphill-Haley, 1993) and flora of north American freshwater species (Patrick and Reimer, 1966; Patrick and Reimer, 1975). Samples were counted by KP and OST, then checked by IS and NB for consistency and comparison with species identified from other sites. Diatom preservation was variable; with some slides giving total counts less than 100 and these levels should be treated with caution. The table below gives the total counted per level, then the percentage frequency of each species for each sample counted. 


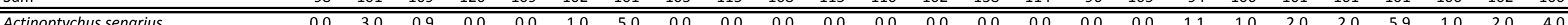

Actinoptychus senarius

Caloneis brevis

Cocconeis peltoides

Delphineis surirella

Diploneis smithii

Gyrosigma wansbeckii

Navicula distans

Navicula palpebralis

Navicula forcipato

Navicula species 1

Nitzschia socialis

Odontella aurita

Paralia sulcata

Pinnularia cruciformis

Plagiogramma vanheurckii

Surirella fastuosa

Thalassionema nitzschioides

Thalassiosira eccentrica

Achnanthes delicatula

Cyclotella striata

Diploneis interrupta

Navicula digitoradiata

Navicula peregrina

Navicula salinarum

Nitzschia obtusa

Nitzschia sigma

Nitzschia vitrea

Rhopalodia operculato

Surirella ovalis

Synedra fasciculata

Amphora veneta

Luticola mutica

Navicula cari var cincto

Nitzschia frustulum

Nitzschia tryblionella

Achnanthes exigua

Achnanthes lanceolata

Amphora ovalis

Caloneis bacillum

Caloneis ventricosa

Ceratoneis arcus

$\begin{array}{lllllllllllllllllllllllll}0.0 & 0.0 & 0.0 & 0.0 & 0.0 & 0.0 & 0.0 & 0.0 & 0.0 & 0.0 & 0.0 & 0.0 & 0.0 & 0.0 & 0.0 & 0.0 & 0.0 & 0.0 & 0.0 & 0.0 & 0.0 & 0.0 & 0.0 & 0.0 & 0.0\end{array}$

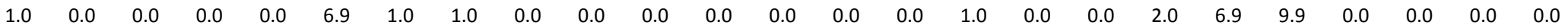

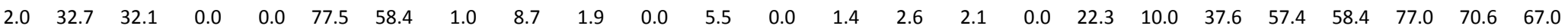

$\begin{array}{lllllllllllllllllllllllll}1.0 & 0.0 & 0.0 & 0.0 & 0.0 & 0.0 & 0.0 & 0.0 & 0.0 & 0.0 & 0.0 & 0.0 & 0.0 & 0.0 & 0.0 & 0.0 & 0.0 & 6.4 & 52.0 & 0.0 & 0.0 & 0.0 & 0.0 & 0.0 & 0.0\end{array}$

$\begin{array}{lllllllllllllllllllllllll}1.0 & 1.0 & 0.0 & 0.0 & 0.0 & 0.0 & 0.0 & 0.0 & 0.0 & 0.0 & 0.0 & 0.0 & 0.0 & 0.0 & 0.0 & 0.0 & 0.0 & 0.0 & 0.0 & 0.0 & 1.0 & 3.0 & 3.0 & 2.0 & 0.0\end{array}$

$\begin{array}{lllllllllllllllllllllllll}0.0 & 0.0 & 0.0 & 0.0 & 0.0 & 0.0 & 1.0 & 0.0 & 0.0 & 0.0 & 0.0 & 0.0 & 0.0 & 0.0 & 0.0 & 0.0 & 0.0 & 0.0 & 0.0 & 0.0 & 0.0 & 0.0 & 0.0 & 0.0 & 0.0\end{array}$

$\begin{array}{lllllllllllllllllllllllll}2.0 & 0.0 & 0.0 & 0.0 & 0.0 & 0.0 & 0.0 & 0.0 & 0.0 & 0.0 & 0.0 & 0.0 & 0.0 & 0.0 & 0.0 & 0.0 & 0.0 & 0.0 & 0.0 & 1.0 & 0.0 & 0.0 & 0.0 & 0.0 & 0.0\end{array}$

$\begin{array}{lllllllllllllllllllllllll}0.0 & 0.0 & 0.0 & 0.0 & 0.0 & 0.0 & 0.0 & 0.0 & 0.0 & 0.0 & 0.0 & 0.0 & 0.0 & 0.0 & 0.0 & 0.0 & 0.0 & 0.0 & 0.0 & 0.0 & 0.0 & 0.0 & 0.0 & 0.0 & 0.0\end{array}$

$\begin{array}{lllllllllllllllllllllllll}2.0 & 0.0 & 0.0 & 0.0 & 0.0 & 0.0 & 0.0 & 0.0 & 0.0 & 0.0 & 0.0 & 0.0 & 0.0 & 0.0 & 0.0 & 0.0 & 0.0 & 0.0 & 2.0 & 0.0 & 0.0 & 0.0 & 0.0 & 0.0 & 0.0\end{array}$

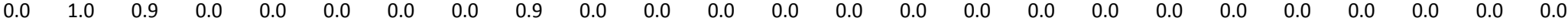

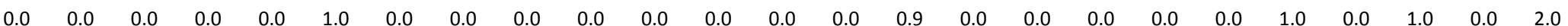

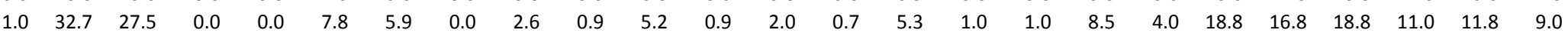

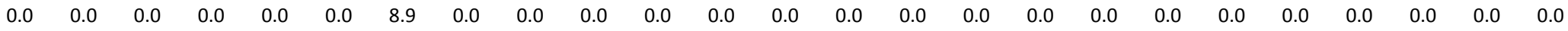

$\begin{array}{lllllllllllllllllllllllll}0.0 & 0.0 & 0.0 & 0.0 & 0.0 & 0.0 & 0.0 & 0.0 & 0.0 & 0.0 & 0.0 & 0.0 & 0.0 & 0.0 & 0.0 & 0.0 & 0.0 & 3.2 & 0.0 & 0.0 & 0.0 & 0.0 & 0.0 & 0.0 & 0.0\end{array}$

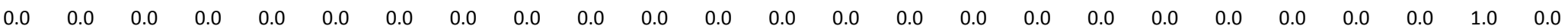

$\begin{array}{lllllllllllllllllllllllll}0.0 & 0.0 & 0.0 & 0.0 & 0.0 & 0.0 & 0.0 & 0.0 & 0.0 & 0.0 & 0.0 & 0.0 & 0.0 & 0.0 & 0.0 & 0.0 & 0.0 & 0.0 & 0.0 & 0.0 & 0.0 & 0.0 & 0.0 & 0.0 & 0.0\end{array}$

$\begin{array}{lllllllllllllllllllllllll}0.0 & 0.0 & 0.9 & 0.0 & 0.0 & 0.0 & 0.0 & 0.0 & 0.0 & 0.0 & 0.0 & 0.0 & 0.0 & 0.0 & 0.0 & 0.0 & 1.0 & 0.0 & 0.0 & 6.9 & 1.0 & 0.0 & 0.0 & 0.0 & 0.0\end{array}$

$\begin{array}{lllllllllllllllllllllllll}0.0 & 0.0 & 0.0 & 0.0 & 0.0 & 0.0 & 0.0 & 0.0 & 0.0 & 0.0 & 0.0 & 0.0 & 0.0 & 0.0 & 0.0 & 0.0 & 0.0 & 0.0 & 0.0 & 0.0 & 0.0 & 0.0 & 0.0 & 0.0 & 0.0\end{array}$

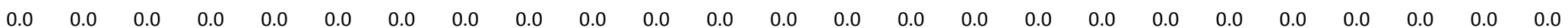

$\begin{array}{lllllllllllllllllllllllll}0.0 & 0.0 & 0.0 & 0.0 & 0.0 & 0.0 & 0.0 & 0.0 & 0.0 & 0.9 & 0.0 & 0.0 & 0.0 & 0.0 & 0.0 & 0.0 & 0.0 & 0.0 & 0.0 & 0.0 & 0.0 & 0.0 & 0.0 & 0.0 & 0.0\end{array}$

$\begin{array}{rrrrrrrrrrrrrrrrrrrrrrrrr}23.5 & 11.9 & 15.6 & 0.0 & 0.0 & 0.0 & 0.0 & 0.0 & 0.0 & 0.0 & 0.0 & 0.0 & 0.0 & 0.0 & 0.0 & 0.0 & 0.0 & 11.7 & 18.0 & 8.9 & 2.0 & 0.0 & 0.0 & 1.0 & 10.0\end{array}$

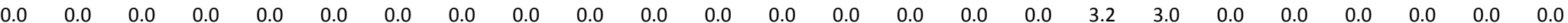

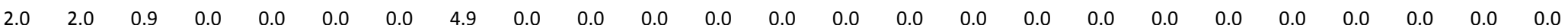

$\begin{array}{lllllllllllllllllllllllll}0.0 & 0.0 & 0.0 & 0.0 & 0.0 & 0.0 & 0.0 & 0.0 & 0.0 & 0.0 & 0.0 & 0.0 & 0.0 & 0.0 & 0.0 & 0.0 & 0.0 & 0.0 & 0.0 & 0.0 & 0.0 & 0.0 & 0.0 & 0.0 & 0.0\end{array}$

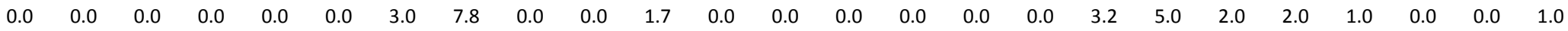

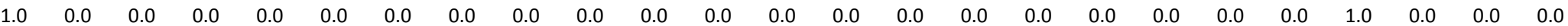

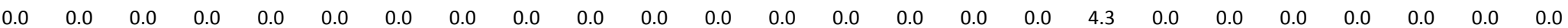

$\begin{array}{lllllllllllllllllllllllll}0.0 & 0.0 & 0.0 & 0.0 & 0.0 & 0.0 & 0.0 & 1.0 & 0.0 & 0.0 & 0.0 & 0.0 & 0.0 & 0.0 & 0.0 & 0.0 & 0.0 & 1.1 & 0.0 & 1.0 & 1.0 & 0.0 & 0.0 & 0.0 & 0.0\end{array}$

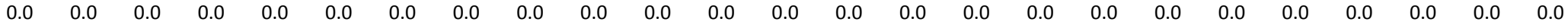

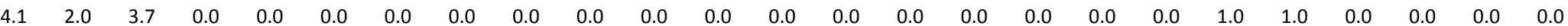

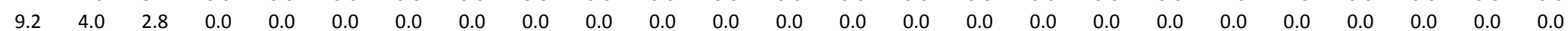

$\begin{array}{llllllllllllllllllllllllll}0.0 & 0.0 & 0.0 & 0.0 & 0.0 & 0.0 & 0.0 & 0.0 & 0.0 & 0.0 & 0.0 & 0.0 & 0.0 & 0.0 & 0.0 & 0.0 & 0.0 & 0.0 & 0.0 & 0.0 & 0.0 & 0.0 & 0.0 & 0.0 & 0.0\end{array}$

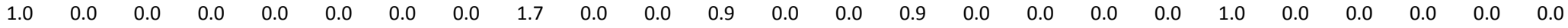

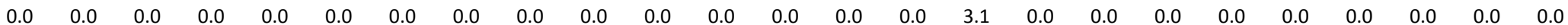

$\begin{array}{llllllllllllllllllllllllll}0.0 & 0.0 & 0.0 & 0.0 & 0.0 & 0.0 & 0.0 & 0.0 & 0.0 & 0.0 & 0.9 & 5.5 & 0.0 & 0.0 & 0.0 & 1.0 & 0.0 & 4.3 & 0.0 & 0.0 & 0.0 & 0.0 & 0.0 & 0.0 & 0.0\end{array}$

$\begin{array}{lllllllllllllllllllllllllll}0.0 & 0.0 & 0.0 & 0.8 & 0.9 & 0.0 & 0.0 & 0.0 & 1.7 & 0.0 & 0.0 & 5.5 & 4.9 & 0.7 & 0.9 & 0.0 & 0.0 & 0.0 & 0.0 & 1.0 & 1.0 & 1.0 & 3.0 & 3.9 & 2.0\end{array}$

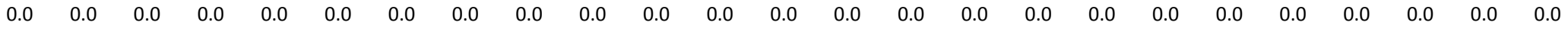

$\begin{array}{lllllllllllllllllllllllll}0.0 & 0.0 & 0.0 & 0.0 & 0.0 & 0.0 & 3.0 & 16.5 & 0.0 & 0.0 & 0.0 & 0.0 & 0.0 & 0.0 & 0.0 & 0.0 & 0.0 & 0.0 & 0.0 & 0.0 & 0.0 & 0.0 & 0.0 & 0.0 & 0.0\end{array}$

$\begin{array}{llllllllllllllllllllllllll}0.0 & 0.0 & 0.0 & 0.0 & 0.0 & 0.0 & 0.0 & 1.9 & 0.0 & 0.0 & 0.0 & 0.0 & 0.0 & 0.0 & 0.0 & 3.1 & 0.0 & 0.0 & 0.0 & 0.0 & 0.0 & 0.0 & 0.0 & 0.0 & 0.0\end{array}$

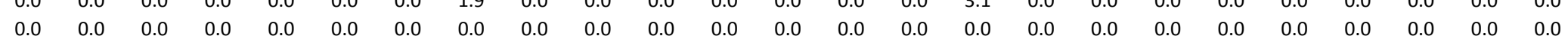


Cymbella affinis

$\begin{array}{llllll}4.1 & 0.0 & 0.0 & 0.0 & 0.0 & 0.0\end{array}$

$\begin{array}{rrrrrrrrrr}0.0 & 0.0 & 0.0 & 0.0 & 0.0 & 0.0 & 0.0 & 0.0 & 0.0 & 0.0 \\ 0.0 & 0.0 & 0.0 & 26.2 & 15.6 & 0.0 & 0.0 & 0.0 & 8.7 & 13.0\end{array}$

0.0
0.0

Cymbella ventricosa

$\begin{array}{lllllllllll}0.0 & 0.0 & 0.0 & 0.0 & 0.0 & 0.0 & 0.0 & 0.0 & 0.0 & 0.0 \\ 0.0 & 26.2 & 15.6 & 0.0 & 0.0 & 0.0 & 8.7 & 13.0 & 10.4 & 11.8\end{array}$

0.00 .0

Diploneis ovalis

Epithemia turgida

Eunotia germaini

Eunotia lunaris

Eunotia pectinalis

Fragilaria construens

Fragilaria vaucheriae

Fragilariforma virescens

Gomphonema acuminatum

Gomphonema gracile

Meridion circulare

Navicula begeri

Navicula brockmanii

Navicula pupula

Navicula pusilla

Navicula radiosa

Navicula tripunctata

Navicula viridula

Nitzschia fruticosa

Nitzschia linearis

Nitzschia palea

Nitzschia palustris

Pinnularia abaujensis

Pinnularia borealis

Pinnularia brevicostata

Pinnularia intermedia

Pinnularia lagerstedtii

Pinnularia mesolepta

Pinnularia microstauron

Pinnularia viridis

Pseudostaurosira brevistriata

$\begin{array}{llllllllllllllllllllllllll}0.0 & 0.0 & 0.0 & 0.0 & 0.0 & 0.0 & 0.0 & 0.0 & 0.0 & 0.0 & 0.0 & 0.0 & 0.0 & 0.0 & 0.0 & 0.0 & 0.0 & 0.0 & 0.0 & 0.0 & 0.0 & 0.0 & 0.0 & 0.0 & 0.0 \\ 0.0 & 1.0 & 0.0 & 0.0 & 0.0 & 0.0 & 0.0 & 0.0 & 0.0 & 0.0 & 0.0 & 0.0 & 0.0 & 0.0 & 0.0 & 0.0 & 0.0 & 0.0 & 0.0 & 0.0 & 0.0 & 0.0 & 0.0 & 0.0 & 0.0\end{array}$

$\begin{array}{lllllllllllllllllllllllll}0.0 & 1.0 & 0.0 & 0.0 & 0.0 & 0.0 & 0.0 & 0.0 & 0.0 & 0.0 & 0.0 & 0.0 & 0.0 & 0.0 & 0.0 & 0.0 & 0.0 & 0.0 & 0.0 & 0.0 & 0.0 & 0.0 & 0.0 & 0.0 & 0.0\end{array}$

$\begin{array}{lllllllllllllllllllllllll}0.0 & 0.0 & 0.0 & 0.0 & 0.0 & 0.0 & 0.0 & 0.0 & 0.0 & 0.0 & 0.0 & 0.0 & 0.0 & 0.0 & 0.0 & 0.0 & 0.0 & 0.0 & 0.0 & 0.0 & 0.0 & 0.0 & 0.0 & 0.0 & 0.0\end{array}$

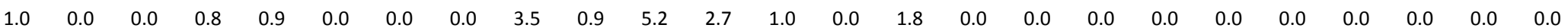

$\begin{array}{llllllllllllllllllllllllll}0.0 & 0.0 & 0.0 & 0.0 & 0.0 & 0.0 & 0.0 & 0.0 & 0.0 & 0.0 & 0.0 & 0.0 & 0.0 & 0.0 & 0.0 & 5.2 & 1.0 & 0.0 & 0.0 & 2.0 & 0.0 & 0.0 & 0.0 & 0.0 & 0.0\end{array}$

$\begin{array}{lllllllllllllllllllllllll}7.1 & 0.0 & 0.0 & 0.8 & 4.6 & 2.9 & 5.0 & 7.8 & 6.1 & 5.6 & 10.4 & 10.0 & 8.8 & 5.8 & 3.5 & 15.6 & 8.6 & 0.0 & 0.0 & 0.0 & 0.0 & 1.0 & 0.0 & 0.0 & 1.0\end{array}$

$\begin{array}{lllllllllllllllllllllllll}0.0 & 0.0 & 0.0 & 0.0 & 0.0 & 0.0 & 0.0 & 0.0 & 0.0 & 0.0 & 0.0 & 2.7 & 2.0 & 2.2 & 0.0 & 0.0 & 0.0 & 0.0 & 0.0 & 0.0 & 0.0 & 0.0 & 0.0 & 0.0 & 0.0\end{array}$

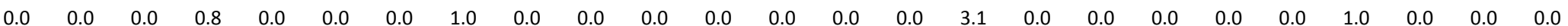

$\begin{array}{lllllllllllllllllllllllll}0.0 & 0.0 & 0.0 & 0.0 & 0.0 & 0.0 & 1.0 & 0.0 & 0.0 & 0.0 & 0.0 & 0.0 & 0.0 & 0.0 & 0.0 & 0.0 & 0.0 & 0.0 & 0.0 & 0.0 & 0.0 & 0.0 & 0.0 & 0.0 & 0.0\end{array}$

$\begin{array}{lllllllllllllllllllllllll}1.0 & 0.0 & 0.0 & 4.0 & 0.0 & 0.0 & 0.0 & 0.0 & 0.0 & 0.0 & 0.0 & 0.0 & 0.0 & 0.7 & 0.0 & 0.0 & 0.0 & 0.0 & 0.0 & 0.0 & 0.0 & 0.0 & 0.0 & 0.0 & 0.0\end{array}$

$\begin{array}{lllllllllllllllllllllllll}0.0 & 0.0 & 0.0 & 0.0 & 0.0 & 0.0 & 0.0 & 0.0 & 0.0 & 0.0 & 0.0 & 0.0 & 0.0 & 0.0 & 0.0 & 0.0 & 0.0 & 0.0 & 0.0 & 0.0 & 0.0 & 0.0 & 0.0 & 0.0 & 0.0\end{array}$

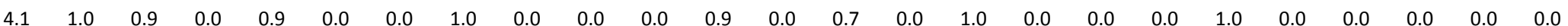

$\begin{array}{llllllllllllllllllllllllll}0.0 & 0.0 & 0.0 & 0.0 & 0.0 & 0.0 & 0.0 & 0.0 & 0.0 & 0.0 & 0.0 & 0.0 & 0.0 & 0.0 & 0.0 & 4.2 & 6.7 & 0.0 & 0.0 & 0.0 & 0.0 & 0.0 & 0.0 & 0.0 & 0.0\end{array}$

$\begin{array}{lllllllllllllllllllllllll}0.0 & 0.0 & 0.0 & 0.0 & 0.0 & 0.0 & 0.0 & 0.0 & 0.0 & 0.0 & 0.0 & 0.0 & 0.0 & 0.0 & 0.0 & 0.0 & 0.0 & 1.1 & 0.0 & 0.0 & 0.0 & 0.0 & 0.0 & 0.0 & 0.0\end{array}$

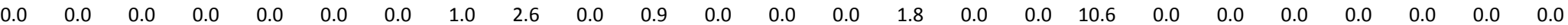

$\begin{array}{lllllllllllllllllllllllll}0.0 & 0.0 & 0.0 & 0.0 & 0.0 & 0.0 & 0.0 & 0.0 & 0.0 & 0.0 & 0.0 & 0.0 & 0.0 & 0.0 & 0.0 & 0.0 & 0.0 & 0.0 & 0.0 & 0.0 & 0.0 & 0.0 & 0.0 & 0.0 & 0.0\end{array}$

$\begin{array}{lllllllllllllllllllllllllll}0.0 & 0.0 & 0.0 & 0.0 & 0.0 & 0.0 & 0.0 & 0.0 & 0.0 & 0.0 & 0.0 & 0.0 & 0.0 & 0.0 & 0.0 & 0.0 & 0.0 & 0.0 & 0.0 & 0.0 & 0.0 & 0.0 & 0.0 & 0.0 & 0.0\end{array}$

$\begin{array}{llllllllllllllllllllllllll}0.0 & 0.0 & 0.0 & 0.0 & 0.0 & 0.0 & 0.0 & 0.0 & 0.0 & 0.0 & 0.0 & 0.0 & 0.0 & 0.0 & 0.0 & 0.0 & 0.0 & 0.0 & 0.0 & 0.0 & 0.0 & 0.0 & 0.0 & 0.0 & 0.0\end{array}$

$\begin{array}{lllllllllllllllllllllllll}0.0 & 0.0 & 0.0 & 0.0 & 0.0 & 0.0 & 0.0 & 0.0 & 0.0 & 0.0 & 0.0 & 0.0 & 0.0 & 0.0 & 0.0 & 0.0 & 0.0 & 0.0 & 0.0 & 0.0 & 0.0 & 0.0 & 0.0 & 0.0 & 0.0\end{array}$

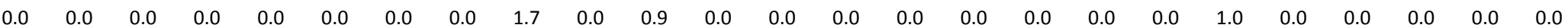

$\begin{array}{llllllllllllllllllllllllll}0.0 & 0.0 & 0.0 & 0.0 & 0.0 & 0.0 & 0.0 & 0.0 & 0.0 & 0.0 & 0.0 & 0.0 & 0.0 & 0.0 & 0.0 & 0.0 & 0.0 & 0.0 & 0.0 & 0.0 & 0.0 & 0.0 & 0.0 & 0.0 & 0.0\end{array}$

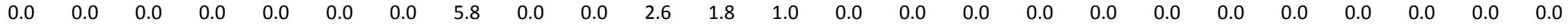

$\begin{array}{lllllllllllllllllllllllll}0.0 & 0.0 & 0.0 & 0.0 & 0.0 & 0.0 & 0.0 & 0.0 & 0.0 & 0.0 & 0.0 & 0.0 & 0.0 & 0.0 & 0.0 & 0.0 & 0.0 & 0.0 & 0.0 & 0.0 & 0.0 & 0.0 & 0.0 & 0.0 & 0.0\end{array}$

$\begin{array}{lllllllllllllllllllllllll}0.0 & 0.0 & 0.0 & 0.0 & 0.0 & 0.0 & 0.0 & 0.0 & 0.0 & 0.0 & 0.0 & 0.0 & 0.0 & 0.0 & 0.0 & 0.0 & 0.0 & 0.0 & 0.0 & 0.0 & 0.0 & 0.0 & 0.0 & 0.0 & 0.0\end{array}$

$\begin{array}{lllllllllllllllllllllllll}0.0 & 0.0 & 0.0 & 0.8 & 0.0 & 0.0 & 0.0 & 0.0 & 0.0 & 0.0 & 0.0 & 0.0 & 0.0 & 0.0 & 0.0 & 0.0 & 0.0 & 0.0 & 0.0 & 0.0 & 0.0 & 0.0 & 0.0 & 0.0 & 0.0\end{array}$

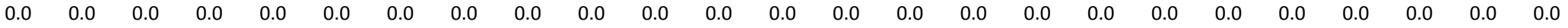

$\begin{array}{lllllllllllllllllllllllll}0.0 & 0.0 & 0.0 & 0.0 & 0.0 & 0.0 & 0.0 & 0.0 & 0.0 & 0.0 & 0.0 & 0.0 & 0.0 & 0.0 & 0.0 & 0.0 & 0.0 & 0.0 & 0.0 & 0.0 & 0.0 & 0.0 & 0.0 & 0.0 & 0.0\end{array}$

$\begin{array}{llllllllllllllllllllllllll}0.0 & 0.0 & 0.0 & 0.0 & 0.0 & 0.0 & 0.0 & 0.0 & 0.0 & 0.0 & 0.0 & 0.0 & 0.0 & 0.0 & 0.0 & 0.0 & 0.0 & 0.0 & 0.0 & 0.0 & 0.0 & 0.0 & 0.0 & 0.0 & 0.0\end{array}$

$\begin{array}{lllllllllllllllllllllllllll}0.0 & 0.0 & 0.0 & 0.8 & 0.0 & 0.0 & 0.0 & 5.8 & 0.9 & 1.9 & 0.0 & 0.0 & 1.0 & 0.7 & 0.0 & 21.9 & 30.5 & 0.0 & 0.0 & 0.0 & 0.0 & 0.0 & 0.0 & 0.0 & 0.0\end{array}$

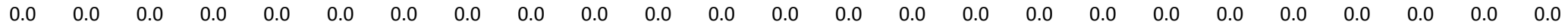

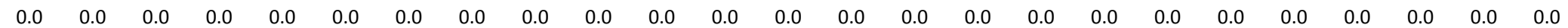

Stauroneis anceps

Stauroneis phoenicentron

Staurosirella pinnato

Synedra ulna

Achnanthes conspicua

Eunotia exigua

$\begin{array}{lllllllllllllllllllllllll}0.0 & 0.0 & 0.0 & 0.0 & 0.0 & 0.0 & 0.0 & 0.0 & 0.0 & 0.0 & 0.0 & 0.0 & 0.0 & 0.0 & 0.0 & 0.0 & 0.0 & 0.0 & 0.0 & 0.0 & 0.0 & 0.0 & 0.0 & 0.0 & 0.0\end{array}$

$\begin{array}{llllllllllllllllllllllllll}0.0 & 0.0 & 0.0 & 0.0 & 0.0 & 0.0 & 0.0 & 0.0 & 0.0 & 0.0 & 0.0 & 0.0 & 0.0 & 0.0 & 0.0 & 0.0 & 0.0 & 0.0 & 0.0 & 0.0 & 3.0 & 0.0 & 0.0 & 0.0 & 0.0\end{array}$

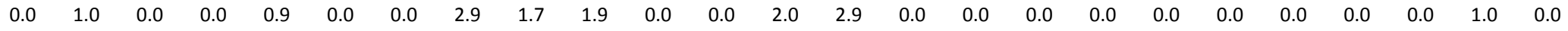

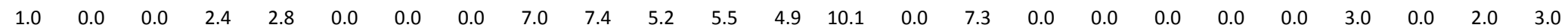

Eunotia praerupto

$\begin{array}{rrrrrrrrrrrrrrrrrrr}1.0 & 0.0 & 1.8 & 31.7 & 26.6 & 0.0 & 5.0 & 0.0 & 8.7 & 13.9 & 10.4 & 14.5 & 3.9 & 15.9 & 11.4 & 0.0 & 0.0 & 0.0 & 0.0 \\ 0.0 & 0.0 & 0.0 & 3.2 & 2.8 & 0.0 & 0.0 & 0.0 & 8.7 & 6.5 & 0.9 & 10.0 & 6.9 & 5.8 & 2.6 & 0.0 & 0.0 & 0.0 & 0.0\end{array}$

$\begin{array}{llllll}0.0 & 0.0 & 0.0 & 0.0 & 0.0 & 0.0\end{array}$

GMC Data Report 404 


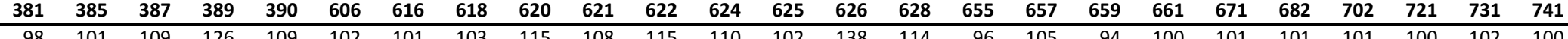

\begin{tabular}{|c|c|c|c|c|c|c|c|c|c|c|c|c|c|c|c|c|c|c|c|c|c|c|c|c|c|}
\hline $\mathrm{m}$ & 98 & 101 & 109 & 126 & 109 & 102 & 101 & 103 & 115 & 108 & 115 & 110 & 102 & 138 & 114 & 96 & 105 & 94 & 100 & 101 & 101 & 101 & 100 & 102 & 100 \\
\hline ail & 0.0 & 0.0 & 0.0 & 0.0 & 0.0 & 0.0 & 0.0 & 0.0 & 0.0 & 0.0 & 0.0 & 0.0 & 0.0 & 0.0 & 0.0 & 0.0 & 0.0 & 0.0 & 0.0 & 0.0 & 0.0 & 0.0 & 0.0 & 0.0 & 0.0 \\
\hline
\end{tabular}

Fragilaria leptostauron

$\begin{array}{lllllll}0.0 & 0.0 & 0.0 & 0.0 & 0.0 & 0.0 & 0.0 \\ 0.0 & 0.0 & 0.0 & 0.0 & 0.0 & 0.0 & 0.0\end{array}$

Nitzschia gracilis

$\begin{array}{lllllllllllllllllll}0.0 & 0.0 & 0.0 & 0.0 & 0.0 & 0.0 & 0.0 & 0.0 & 0.0 & 0.0 & 0.0 & 0.0 & 0.0 & 0.0 & 0.0 & 0.0 & 0.0 & 0.0 & 0.0\end{array}$

Pinnularia subcapitato

$\begin{array}{lllllll}0.0 & 0.0 & 0.0 & 0.0 & 0.0 & 0.0 & 0.0 \\ 0.0 & 0.0 & 0.0 & 0.0 & 0.0 & 2.0 & 3.0\end{array}$

Pinnularia subsolaris

Tabellaria fenestrata

Tabellaria flocculosa

Navicula ramosissima

Neidium bisulcatum

Cyclotella meneghiniana

Caloneis westii

$\begin{array}{llllllllllllllllllllllllll}0.0 & 0.0 & 0.0 & 0.0 & 0.0 & 2.0 & 3.0 & 8.7 & 0.9 & 5.6 & 5.2 & 0.9 & 2.9 & 2.2 & 0.0 & 0.0 & 3.8 & 4.3 & 0.0 & 0.0 & 0.0 & 0.0 & 0.0 & 0.0 & 1.0 \\ 0.0 & 0.0 & 0.0 & 0.0 & 0.0 & 0.0 & 0.0 & 1.0 & 0.0 & 0.0 & 0.0 & 0.0 & 0.0 & 0.0 & 0.0 & 0.0 & 0.0 & 0.0 & 0.0 & 0.0 & 0.0 & 0.0 & 0.0 & 0.0 & 0.0\end{array}$

$\begin{array}{lllllllllllllllllllllllll}0.0 & 0.0 & 0.9 & 0.0 & 0.0 & 0.0 & 0.0 & 0.0 & 0.0 & 0.0 & 0.0 & 0.0 & 0.0 & 0.0 & 0.0 & 0.0 & 0.0 & 0.0 & 0.0 & 0.0 & 0.0 & 0.0 & 0.0 & 0.0 & 0.0\end{array}$

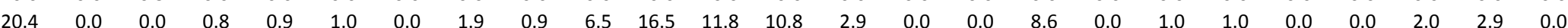

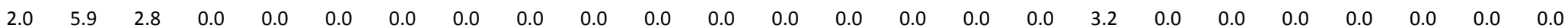

$\begin{array}{lllllllllllllllllllllllll}0.0 & 0.0 & 0.0 & 0.0 & 0.0 & 0.0 & 0.0 & 0.0 & 0.0 & 0.0 & 0.0 & 0.0 & 0.0 & 0.0 & 0.0 & 0.0 & 0.0 & 0.0 & 0.0 & 0.0 & 0.0 & 0.0 & 0.0 & 0.0 & 0.0\end{array}$

Eunotia tenella

Fragilaria construens var. binodis

Navicula capitata var. hungarica

Pinnularia major

Aulacoseira distans

Navicula subtilissima

Fragilariforma constricta

Achnanthes clevei

Cyclotella radiosa

Fragilaria construens var. venter

Navicula ignota var. palustris

$\begin{array}{llllllllllllllllllllllllll}2.0 & 0.0 & 0.0 & 0.0 & 1.8 & 0.0 & 0.0 & 0.0 & 0.0 & 0.0 & 0.0 & 0.0 & 0.0 & 0.7 & 0.9 & 1.0 & 0.0 & 0.0 & 0.0 & 0.0 & 0.0 & 1.0 & 2.0 & 1.0 & 0.0\end{array}$

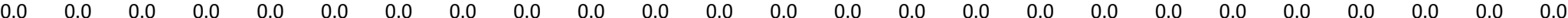

$\begin{array}{lllllllllllllllllllllllll}0.0 & 0.0 & 4.6 & 16.7 & 30.3 & 0.0 & 0.0 & 2.9 & 20.9 & 16.7 & 6.1 & 3.6 & 9.8 & 15.2 & 10.5 & 0.0 & 0.0 & 0.0 & 0.0 & 0.0 & 0.0 & 0.0 & 0.0 & 0.0 & 0.0\end{array}$

$\begin{array}{lllllllllllllllllllllllll}0.0 & 0.0 & 0.0 & 0.0 & 0.0 & 0.0 & 0.0 & 0.0 & 0.0 & 0.0 & 0.0 & 0.0 & 0.0 & 0.0 & 0.0 & 0.0 & 0.0 & 0.0 & 0.0 & 0.0 & 0.0 & 0.0 & 0.0 & 0.0 & 0.0\end{array}$

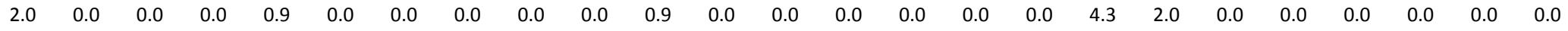

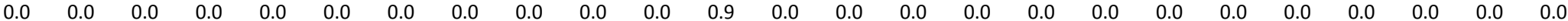

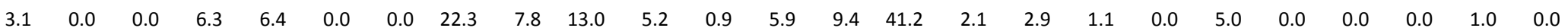

$\begin{array}{llllllllllllllllllllllllll}0.0 & 0.0 & 0.0 & 0.8 & 0.0 & 0.0 & 0.0 & 0.0 & 0.0 & 0.0 & 0.0 & 0.0 & 0.0 & 0.0 & 0.0 & 0.0 & 0.0 & 0.0 & 0.0 & 0.0 & 0.0 & 0.0 & 0.0 & 0.0 & 0.0\end{array}$

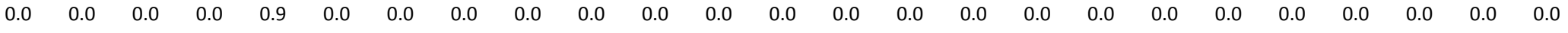

$\begin{array}{llllllllllllllllllllllllll}0.0 & 0.0 & 0.0 & 0.0 & 0.0 & 0.0 & 0.0 & 0.0 & 0.0 & 0.0 & 0.0 & 0.0 & 0.0 & 0.0 & 0.0 & 0.0 & 0.0 & 0.0 & 0.0 & 0.0 & 0.0 & 0.0 & 0.0 & 0.0 & 0.0\end{array}$

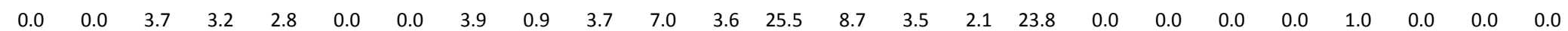

$\begin{array}{lllllllllllllllllllllllll}0.0 & 0.0 & 0.0 & 0.0 & 0.0 & 0.0 & 0.0 & 0.0 & 0.0 & 0.0 & 0.0 & 0.0 & 0.0 & 0.0 & 0.0 & 0.0 & 0.0 & 0.0 & 0.0 & 0.0 & 0.0 & 0.0 & 0.0 & 0.0 & 0.0\end{array}$

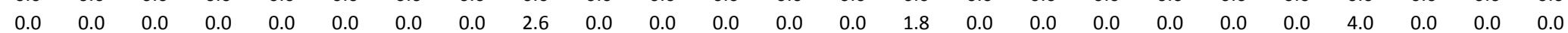




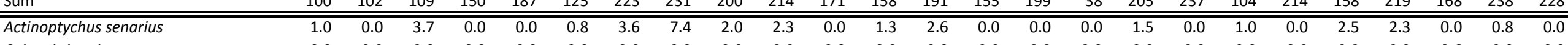

Actinoptychus senarius

Caloneis brevis

Cocconeis peltoides

$\begin{array}{llllllllllllllll}0.0 & 0.0 & 0.0 & 0.0 & 0.0 & 0.0 & 0.0 & 0.0 & 0.0 & 0.0 & 0.0 & 0.0 & 0.0 & 0.0 & 0.0 & 0.0\end{array}$

Delphineis surirella

Diploneis smithii

$\begin{array}{llllllllllllllllllllllllll}4.0 & 4.9 & 0.0 & 0.0 & 0.0 & 0.0 & 0.0 & 4.3 & 6.0 & 1.9 & 0.0 & 0.0 & 1.0 & 0.0 & 0.0 & 0.0 & 0.0 & 0.0 & 0.0 & 0.0 & 0.0 & 3.2 & 0.0 & 0.0 & 0.9\end{array}$

Gyrosigma wansbeckii

$\begin{array}{llllllllllllllllllll}85.0 & 73.5 & 50.5 & 2.7 & 0.0 & 1.6 & 53.8 & 49.8 & 60.0 & 56.1 & 52.6 & 70.3 & 34.0 & 20.6 & 15.1 & 97.4 & 6.8 & 1.7 & 28.8 & 4.7\end{array}$

Navicula distans

$\begin{array}{lllllllllllllllllllllllllll}0.0 & 0.0 & 0.0 & 0.0 & 0.0 & 0.0 & 0.0 & 0.9 & 1.0 & 0.5 & 0.0 & 0.0 & 0.0 & 0.0 & 0.0 & 0.0 & 0.0 & 0.0 & 0.0 & 0.0 & 0.0 & 1.4 & 0.0 & 0.0 & 0.0\end{array}$

Navicula

$\begin{array}{rrrrrrrrrrrrrrrrrrrrrrrrr}2.0 & 2.9 & 0.0 & 0.0 & 0.0 & 0.0 & 0.0 & 0.4 & 2.5 & 2.8 & 19.9 & 7.6 & 0.0 & 0.0 & 0.0 & 0.0 & 0.0 & 0.0 & 0.0 & 0.0 & 0.0 & 0.9 & 1.2 & 0.0 & 1.8 \\ 0.0 & 0.0 & 0.0 & 0.0 & 0.0 & 0.0 & 0.0 & 0.0 & 0.0 & 0.0 & 0.0 & 0.0 & 0.0 & 0.0 & 0.0 & 0.0 & 0.0 & 0.0 & 0.0 & 0.0 & 0.0 & 0.0 & 0.0 & 0.0 & 0.0 \\ 0.0 & 0.0 & 0.0 & 0.0 & 0.0 & 0.0 & 0.0 & 0.0 & 0.0 & 0.0 & 0.0 & 0.0 & 0.0 & 0.0 & 0.0 & 0.0 & 0.0 & 0.0 & 0.0 & 0.0 & 0.0 & 0.0 & 0.0 & 0.0 & 0.0\end{array}$

Navicula palpebralis

Navicula species 1

Nitzschia socialis

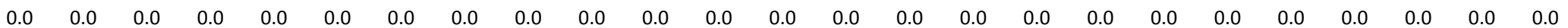

$\begin{array}{lllllllllllllllllllllllll}0.0 & 0.0 & 0.0 & 0.0 & 0.0 & 0.0 & 0.9 & 0.0 & 0.0 & 0.0 & 0.0 & 2.5 & 0.0 & 0.0 & 0.0 & 0.0 & 0.0 & 0.0 & 0.0 & 0.0 & 0.0 & 0.5 & 4.8 & 1.7 & 11.0\end{array}$

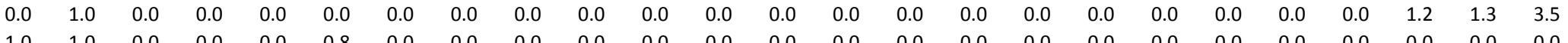

$\begin{array}{llllllllllllllllllllllllll}1.0 & 1.0 & 0.0 & 0.0 & 0.0 & 0.8 & 0.0 & 0.0 & 0.0 & 0.0 & 0.0 & 0.0 & 0.0 & 0.0 & 0.0 & 0.0 & 0.0 & 0.0 & 0.0 & 0.0 & 0.0 & 0.0 & 0.0 & 0.0 & 0.0\end{array}$

Odontella aurita

$\begin{array}{lllllllllllllllllllllllll}0.0 & 0.0 & 0.0 & 0.0 & 0.0 & 0.0 & 0.0 & 0.0 & 0.0 & 0.0 & 0.0 & 0.0 & 1.6 & 0.0 & 0.0 & 0.0 & 0.0 & 0.0 & 0.0 & 0.0 & 0.6 & 0.0 & 0.0 & 0.0 & 0.0\end{array}$

Paralia sulcata

Pinnularia cruciformis

Plagiogramma vanheurckii

Surirella fastuosa

Thalassionema nitzschioides

Thalassiosira eccentrica

Achnanthes delicatula

Cyclotella striata

Diploneis interrupta

Navicula digitoradiata

Navicula peregrina

Navicula salinarum

Nitzschia obtusa

Nitzschia sigma

Nitzschia vitrea

Rhopalodia operculata

Surirella ovalis

Synedra fasciculata

Amphora veneta

Luticola mutica

Navicula cari var cincto

Nitzschia frustulum

Nitzschia tryblionella

Achnanthes exigua

Achnanthes lanceolata

Amphora ovalis

$\begin{array}{llllllllllllllll}1.0 & 5.9 & 33.0 & 0.0 & 0.0 & 0.0 & 23.8 & 19.5 & 4.5 & 18.7 & 11.7 & 5.7 & 24.1 & 0.0 & 0.0\end{array}$

$\begin{array}{lllllllllllllllllllllllll}0.0 & 0.0 & 0.0 & 0.0 & 0.0 & 0.0 & 0.0 & 0.0 & 0.0 & 0.0 & 0.0 & 0.0 & 0.0 & 0.0 & 0.0 & 0.0 & 0.0 & 0.0 & 0.0 & 0.0 & 0.0 & 0.0 & 0.0 & 0.0 & 0.0\end{array}$

$\begin{array}{lllllllllllllllllllllllll}0.0 & 0.0 & 0.0 & 0.0 & 0.0 & 0.0 & 0.0 & 0.0 & 0.0 & 0.0 & 0.0 & 0.0 & 0.0 & 0.0 & 0.0 & 0.0 & 0.0 & 0.0 & 0.0 & 0.0 & 0.0 & 0.0 & 0.0 & 0.0 & 0.0\end{array}$

$\begin{array}{lllllllllllllllllllllllll}0.0 & 0.0 & 0.0 & 0.0 & 0.0 & 0.0 & 0.0 & 0.4 & 0.0 & 0.0 & 0.0 & 0.0 & 0.0 & 0.0 & 0.0 & 0.0 & 0.0 & 0.0 & 0.0 & 0.0 & 0.0 & 0.0 & 0.0 & 0.0 & 0.0\end{array}$

$\begin{array}{llllllllllllllllllllllllll}0.0 & 0.0 & 0.0 & 0.0 & 0.0 & 0.0 & 0.4 & 0.4 & 1.5 & 0.5 & 0.0 & 1.3 & 3.1 & 0.0 & 0.0 & 0.0 & 0.0 & 0.8 & 1.0 & 0.0 & 2.5 & 0.5 & 0.6 & 0.0 & 0.0\end{array}$

$\begin{array}{lllllllllllllllllllllllll}0.0 & 1.0 & 0.0 & 0.0 & 0.0 & 0.0 & 0.0 & 3.0 & 3.0 & 0.5 & 0.0 & 0.0 & 2.6 & 0.0 & 0.0 & 0.0 & 0.0 & 0.0 & 2.9 & 0.0 & 0.0 & 0.9 & 0.0 & 0.0 & 0.0\end{array}$

$\begin{array}{lllllllllllllllllllllllll}0.0 & 1.0 & 0.0 & 0.0 & 0.0 & 0.0 & 0.0 & 3.0 & 0.0 & 1.9 & 0.0 & 0.0 & 7.9 & 0.0 & 0.0 & 0.0 & 0.0 & 0.0 & 1.9 & 0.0 & 3.2 & 0.0 & 0.0 & 0.0 & 0.0\end{array}$

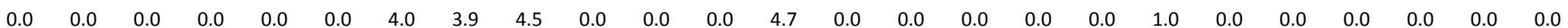

$\begin{array}{llllllllllllllllllllllllll}0.0 & 0.0 & 0.0 & 0.0 & 0.0 & 0.0 & 0.0 & 0.0 & 0.0 & 0.0 & 0.0 & 0.0 & 0.0 & 0.0 & 0.0 & 0.0 & 0.0 & 0.0 & 0.0 & 0.0 & 0.0 & 0.0 & 0.0 & 0.0 & 0.0\end{array}$

$\begin{array}{llllllllllllllllllllllllll}0.0 & 0.0 & 0.9 & 0.0 & 0.0 & 0.8 & 0.0 & 0.0 & 0.0 & 0.0 & 0.0 & 0.0 & 0.0 & 0.0 & 0.0 & 0.0 & 0.0 & 0.0 & 0.0 & 0.0 & 0.0 & 0.0 & 0.0 & 0.0 & 0.0\end{array}$

$\begin{array}{llllllllllll}0.0 & 0.0 & 0.0 & 0.0 & 0.0 & 0.0 & 0.9 & 2.2 & 3.0 & 4.7 & 0.0 & 1.3\end{array}$

$\begin{array}{lllllllllllllllllllllllllllll}0.0 & 1.0 & 0.0 & 0.0 & 0.0 & 0.0 & 0.0 & 0.0 & 0.0 & 0.0 & 0.0 & 0.0 & 0.0 & 0.0 & 0.0 & 0.0 & 0.0 & 0.0 & 0.0 & 0.0 & 0.0 & 0.0 & 0.0 & 0.0 & 0.0 \\ 0.0 & 0.0 & 0.0 & 0.0 & 0.0 & 0.0 & 0.0 & 0.0 & 1.5 & 0.0 & 0.0 & 0.0 & 0.0 & 0.0 & 0.0 & 0.0 & 0.0 & 0.0 & 1.0 & 2.3 & 0.0 & 0.0 & 0.0 & 0.0 & 0.0\end{array}$

$\begin{array}{rllllllllllllllllllllllll}0.0 & 0.0 & 0.0 & 0.0 & 0.0 & 0.0 & 0.0 & 0.0 & 2.0 & 1.4 & 0.0 & 0.0 & 2.1 & 0.0 & 0.0 & 0.0 & 0.0 & 0.0 & 1.9 & 10.3 & 1.9 & 0.9 & 11.9 & 5.5 & 0.0\end{array}$

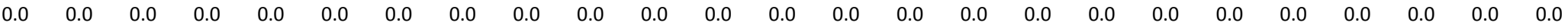

$\begin{array}{lllllllllllllllllllllllll}0.0 & 0.0 & 0.0 & 0.0 & 0.0 & 0.0 & 0.0 & 0.0 & 0.0 & 0.0 & 0.0 & 0.0 & 0.0 & 0.0 & 0.0 & 0.0 & 0.0 & 0.0 & 0.0 & 0.0 & 0.0 & 0.0 & 0.0 & 0.8 & 0.0\end{array}$

$\begin{array}{llllllllllllllllllllllllllll}0.0 & 0.0 & 0.9 & 0.0 & 0.0 & 0.0 & 0.9 & 0.0 & 0.0 & 0.0 & 0.0 & 2.5 & 0.0 & 0.0 & 0.0 & 0.0 & 0.5 & 0.0 & 0.0 & 0.0 & 0.0 & 0.0 & 0.0 & 0.0 & 0.0\end{array}$

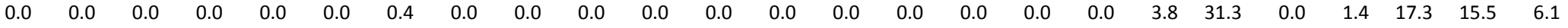

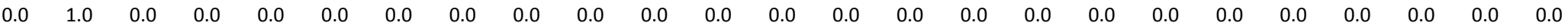

$\begin{array}{lllllllllllllllllllllllll}0.0 & 2.0 & 4.6 & 0.0 & 0.0 & 0.0 & 0.0 & 0.0 & 0.0 & 0.0 & 0.0 & 0.0 & 0.0 & 0.0 & 0.0 & 0.0 & 0.0 & 0.0 & 0.0 & 0.0 & 0.0 & 0.0 & 0.0 & 0.0 & 0.0\end{array}$

$\begin{array}{lllllllllllllllllllllllll}0.0 & 0.0 & 0.0 & 0.0 & 0.0 & 0.0 & 1.8 & 0.0 & 0.0 & 0.0 & 0.6 & 0.6 & 0.0 & 0.0 & 0.0 & 0.0 & 0.0 & 0.0 & 0.0 & 0.0 & 0.0 & 0.0 & 2.4 & 0.0 & 13.6\end{array}$

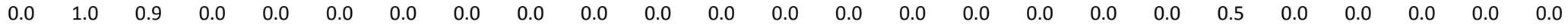

$\begin{array}{lllllllllllllllllllllllll}0.0 & 0.0 & 0.0 & 0.0 & 0.0 & 0.0 & 0.0 & 0.0 & 0.0 & 0.0 & 0.0 & 0.0 & 0.0 & 0.0 & 0.0 & 0.0 & 0.0 & 0.0 & 0.0 & 0.0 & 0.0 & 0.0 & 0.0 & 0.0 & 0.0\end{array}$

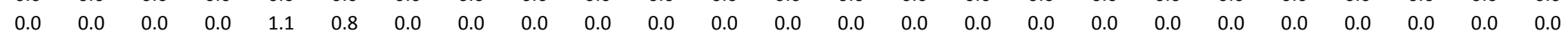

$\begin{array}{llllllllllllllllllllllllll}0.0 & 0.0 & 0.0 & 0.0 & 0.0 & 0.8 & 0.0 & 0.0 & 0.0 & 0.0 & 0.0 & 0.0 & 0.0 & 0.0 & 0.0 & 0.0 & 0.0 & 0.0 & 0.0 & 0.0 & 0.0 & 0.0 & 0.0 & 0.0 & 0.0\end{array}$

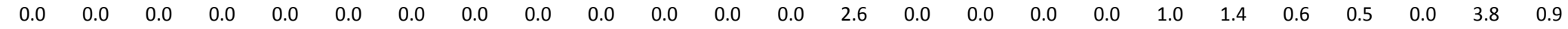

$\begin{array}{lllllllllllllllllllllllll}0.0 & 0.0 & 0.0 & 0.0 & 0.0 & 0.0 & 0.0 & 0.0 & 0.0 & 0.0 & 0.0 & 0.0 & 0.0 & 0.0 & 0.0 & 0.0 & 0.0 & 0.0 & 0.0 & 0.0 & 0.0 & 0.5 & 0.0 & 0.0 & 0.0\end{array}$

Caloneis bacillum

Caloneis ventricosa

Ceratoneis arcus

$\begin{array}{llllllllllll}0.0 & 0.0 & 0.0 & 0.0 & 0.0 & 0.0 & 0.0 & 0.0 & 0.0 & 0.0 & 0.0 & 0.0 \\ 0.0 & 0.0 & 0.0 & 0.0 & 0.0 & 0.0 & 0.0 & 0.0 & 0.0 & 0.0 & 0.0 & 0.0\end{array}$

$\begin{array}{llll}.0 & 0.0 & 0.0 & 0.0 \\ 0.0 & 0.0 & 0.0 & 0.0\end{array}$

$0.0 \quad 0.0$

$\begin{array}{lll}0.0 & 0.0 & 0.0\end{array}$

$\begin{array}{lllll}0.0 & 0.0 & 0.0 & 0.0 & 0.0\end{array}$

GMC Data Report 404 
Cymbella affinis

$\begin{array}{llllllllll}0.0 & 0.0 & 0.0 & 0.0 & 0.5 & 0.0 & 0.0 & 0.0 & 0.0 & 0.0\end{array}$

$\begin{array}{rrrrrrrrrrrrrrrrrrrrrrrrr}0.0 & 0.0 & 0.0 & 0.0 & 0.0 & 0.0 & 0.0 & 0.0 & 0.0 & 0.0 & 0.0 & 0.0 & 0.0 & 0.0 & 0.0 & 0.0 & 0.0 & 0.0 & 0.0 & 0.0 & 0.6 & 0.5 & 0.0 & 0.0 & 0.0 \\ 0.0 & 0.0 & 0.9 & 30.0 & 23.5 & 12.8 & 0.0 & 0.0 & 0.0 & 0.0 & 0.0 & 0.0 & 0.0 & 0.0 & 0.0 & 0.0 & 0.0 & 0.0 & 0.0 & 0.5 & 0.0 & 0.0 & 0.0 & 0.0 & 0.0\end{array}$

Cymbella ventricosa

Diploneis ovalis

Epithemia turgida

Eunotia germaini

Eunotia lunaris

Eunotia pectinalis

Fragilaria construens

Fragilaria vaucheriae

Fragilariforma virescens

Gomphonema acuminatum

Gomphonema gracile

Meridion circulare

Navicula begeri

Navicula brockmanii

Navicula pupula

Navicula pusilla

Navicula radiosa

Navicula tripunctata

Navicula viridula

Nitzschia fruticosa

Nitzschia linearis

Nitzschia palea

Nitzschia palustris

Pinnularia abaujensis

Pinnularia borealis

Pinnularia brevicostata

Pinnularia intermedia

Pinnularia lagerstedtii

Pinnularia mesolepta

Pinnularia microstauron

Pinnularia viridis

Pseudostaurosira brevistriata

$\begin{array}{llllllllllll}0.0 & 0.0 & 0.0 & 0.0 & 0.0 & 0.5 & 0.0 & 0.0 & 0.0 & 0.0 & 0.0\end{array}$

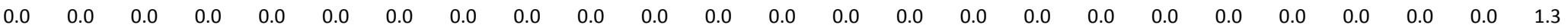

$\begin{array}{lllllllllllllllllllllllll}0.0 & 0.0 & 0.0 & 0.0 & 0.0 & 0.0 & 0.4 & 0.0 & 0.0 & 0.0 & 0.0 & 0.0 & 0.0 & 0.6 & 0.0 & 0.0 & 0.0 & 0.4 & 0.0 & 0.0 & 0.6 & 0.9 & 0.0 & 0.0 & 0.0\end{array}$

$\begin{array}{rrrrrrrrrrrrrrrrrrrrrrrrrrrr}0.0 & 0.0 & 0.0 & 0.0 & 0.0 & 0.0 & 0.0 & 0.0 & 0.0 & 0.0 & 0.0 & 0.0 & 0.0 & 0.0 & 0.0 & 0.0 & 0.0 & 0.0 & 0.0 & 0.0 & 0.0 & 4.1 & 0.0 & 0.0 & 0.0\end{array}$

$\begin{array}{lllllllllllllllllllllllll}0.0 & 0.0 & 0.0 & 0.0 & 0.0 & 0.0 & 0.0 & 0.0 & 1.0 & 0.0 & 0.0 & 0.0 & 0.0 & 16.1 & 16.6 & 0.0 & 15.1 & 5.1 & 7.7 & 7.9 & 0.0 & 6.4 & 0.6 & 0.0 & 0.0\end{array}$

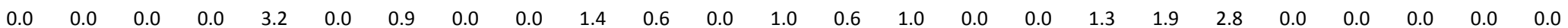

$\begin{array}{llllllllllllllllllllllllll}0.0 & 0.0 & 0.0 & 0.0 & 0.0 & 0.0 & 0.0 & 0.0 & 0.0 & 0.0 & 0.0 & 0.0 & 0.0 & 0.0 & 0.0 & 0.0 & 0.0 & 0.0 & 0.0 & 0.0 & 0.0 & 0.0 & 0.0 & 0.0 & 0.0\end{array}$

$\begin{array}{lllllllllllllllllllllllll}3.0 & 2.0 & 0.9 & 0.0 & 0.0 & 0.0 & 0.0 & 0.0 & 0.0 & 0.0 & 0.0 & 0.0 & 0.0 & 0.0 & 0.0 & 0.0 & 0.0 & 0.0 & 0.0 & 0.0 & 0.0 & 0.0 & 0.0 & 0.0 & 0.0\end{array}$

$\begin{array}{llllllllllllllllllllllllll}0.0 & 0.0 & 0.0 & 0.0 & 7.0 & 0.0 & 0.0 & 0.0 & 0.0 & 0.0 & 0.0 & 0.0 & 0.0 & 0.0 & 0.0 & 0.0 & 0.0 & 0.0 & 0.0 & 0.0 & 0.0 & 0.0 & 0.0 & 0.0 & 0.0\end{array}$

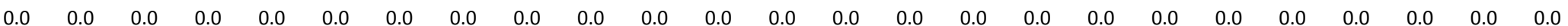

$\begin{array}{lllllllllllllllllllllllll}0.0 & 0.0 & 0.0 & 0.0 & 0.0 & 0.0 & 0.0 & 1.3 & 0.0 & 0.0 & 0.0 & 0.0 & 0.0 & 0.0 & 0.0 & 0.0 & 0.0 & 0.0 & 0.0 & 0.0 & 0.0 & 1.4 & 0.0 & 0.0 & 0.0\end{array}$

$\begin{array}{lllllllllllllllllllllllll}0.0 & 0.0 & 0.0 & 0.0 & 0.0 & 0.0 & 0.0 & 0.0 & 0.0 & 0.0 & 0.0 & 0.0 & 0.0 & 0.0 & 0.0 & 0.0 & 0.0 & 0.0 & 0.0 & 0.0 & 0.0 & 0.0 & 0.0 & 0.0 & 0.0\end{array}$

$\begin{array}{lllllllllllllllllllllllll}0.0 & 0.0 & 0.0 & 0.7 & 0.0 & 0.0 & 0.0 & 0.0 & 0.0 & 0.0 & 0.0 & 0.0 & 0.0 & 0.0 & 0.0 & 0.0 & 0.0 & 0.0 & 0.0 & 0.0 & 0.0 & 0.0 & 0.0 & 0.0 & 0.0\end{array}$

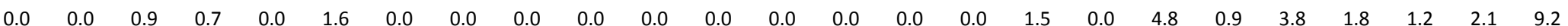

$\begin{array}{lllllllllllllllllllllllll}0.0 & 0.0 & 0.0 & 0.0 & 0.0 & 0.0 & 0.0 & 0.0 & 0.5 & 0.9 & 0.0 & 0.6 & 0.5 & 0.0 & 2.0 & 0.0 & 0.0 & 0.0 & 0.0 & 0.0 & 0.0 & 0.5 & 0.0 & 0.4 & 1.3\end{array}$

$\begin{array}{lllllllllllllllllllllllll}0.0 & 0.0 & 0.0 & 0.0 & 0.0 & 0.0 & 0.0 & 0.0 & 0.0 & 0.0 & 0.0 & 0.0 & 0.0 & 0.0 & 0.0 & 0.0 & 0.0 & 0.0 & 0.0 & 0.0 & 0.0 & 0.9 & 2.4 & 2.1 & 1.8\end{array}$

$\begin{array}{lllllllllllllllllllllllll}0.0 & 0.0 & 0.0 & 0.7 & 0.0 & 2.4 & 0.0 & 0.0 & 0.0 & 0.0 & 0.0 & 0.0 & 0.0 & 0.0 & 0.0 & 0.0 & 0.0 & 0.0 & 0.0 & 0.0 & 0.0 & 0.0 & 0.0 & 0.0 & 0.0\end{array}$

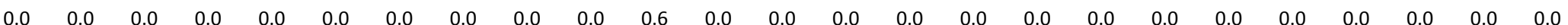

$\begin{array}{lllllllllllllllllllllllll}0.0 & 0.0 & 0.0 & 0.0 & 0.0 & 0.0 & 0.0 & 0.0 & 0.0 & 0.0 & 0.0 & 0.0 & 0.0 & 0.0 & 0.0 & 0.0 & 0.0 & 0.0 & 0.0 & 0.0 & 0.6 & 0.0 & 0.0 & 0.0 & 0.9\end{array}$

$\begin{array}{llllllllllllllllllllllllll}0.0 & 0.0 & 0.0 & 0.0 & 0.0 & 0.0 & 0.0 & 0.0 & 0.0 & 0.0 & 0.0 & 0.0 & 0.0 & 0.0 & 0.0 & 0.0 & 0.0 & 0.0 & 0.0 & 0.9 & 0.6 & 0.0 & 1.2 & 1.3 & 2.6\end{array}$

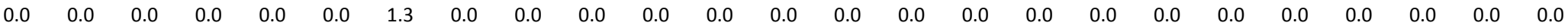

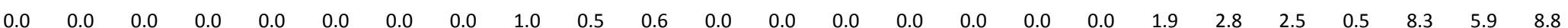

$\begin{array}{lllllllllllllllllllllllllll}0.0 & 0.0 & 0.0 & 0.0 & 0.0 & 0.0 & 0.0 & 0.0 & 0.0 & 0.0 & 0.0 & 0.0 & 0.0 & 0.0 & 0.0 & 0.0 & 0.0 & 0.0 & 0.0 & 0.0 & 0.0 & 0.9 & 0.0 & 2.5 & 0.0\end{array}$

$\begin{array}{lllllllllllllllllllllllll}0.0 & 0.0 & 0.0 & 0.0 & 0.0 & 2.4 & 0.0 & 0.0 & 0.0 & 0.0 & 0.0 & 0.0 & 0.0 & 0.0 & 0.0 & 0.0 & 0.0 & 0.0 & 0.0 & 0.0 & 0.0 & 0.0 & 0.0 & 0.0 & 0.0\end{array}$

$\begin{array}{lllllllllllllllllllllllll}0.0 & 0.0 & 0.0 & 0.0 & 0.0 & 0.0 & 0.0 & 0.0 & 0.0 & 0.0 & 0.0 & 0.6 & 0.0 & 0.0 & 0.0 & 0.0 & 0.0 & 0.0 & 1.0 & 0.5 & 3.8 & 3.2 & 1.8 & 2.1 & 8.8\end{array}$

$\begin{array}{lllllllllllllllllllllllll}0.0 & 0.0 & 0.0 & 0.0 & 0.0 & 0.0 & 0.0 & 0.0 & 0.0 & 0.0 & 2.3 & 0.0 & 0.0 & 0.0 & 0.0 & 0.0 & 3.4 & 22.8 & 1.0 & 3.3 & 0.0 & 2.3 & 0.0 & 0.0 & 0.4\end{array}$

$\begin{array}{lllllllllllllllllllllllll}0.0 & 0.0 & 0.0 & 0.0 & 0.0 & 0.8 & 0.0 & 0.0 & 0.0 & 0.0 & 0.0 & 0.0 & 0.0 & 0.0 & 0.0 & 0.0 & 0.0 & 0.0 & 0.0 & 0.0 & 0.0 & 0.0 & 0.0 & 0.0 & 0.0\end{array}$

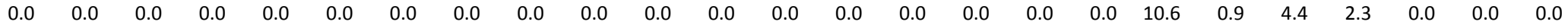

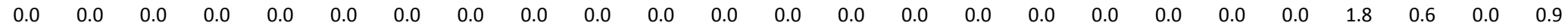

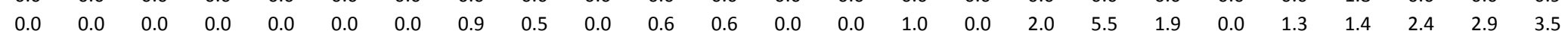

$\begin{array}{rrrrrrrrrrrrrrrrrrrrrrrrrrr}0.0 & 0.0 & 0.0 & 0.0 & 3.2 & 15.2 & 1.3 & 0.0 & 0.0 & 0.5 & 1.2 & 1.3 & 0.0 & 0.0 & 0.5 & 0.0 & 5.4 & 24.9 & 7.7 & 10.7 & 8.9 & 8.2 & 1.2 & 13.9 & 1.8\end{array}$

$\begin{array}{lllllllllllllllllllllllll}1.0 & 0.0 & 0.0 & 0.0 & 0.0 & 0.0 & 0.0 & 0.0 & 0.0 & 0.0 & 0.0 & 0.0 & 0.0 & 0.0 & 0.0 & 0.0 & 0.0 & 0.0 & 0.0 & 0.0 & 0.0 & 0.0 & 0.0 & 0.0 & 0.0\end{array}$

$\begin{array}{lllllllllllllllllllllllll}0.0 & 0.0 & 0.0 & 0.0 & 0.0 & 0.0 & 0.0 & 0.0 & 0.0 & 0.0 & 0.0 & 0.6 & 0.0 & 0.0 & 0.0 & 0.0 & 0.0 & 0.0 & 0.0 & 3.3 & 1.3 & 0.5 & 4.2 & 1.3 & 2.6\end{array}$

Stauroneis anceps

Stauroneis phoenicentron

Staurosirella pinnato

Synedra ulna

Achnanthes conspicua

Eunotia exigua

$\begin{array}{lllllllllllllllllllllllll}0.0 & 0.0 & 0.0 & 0.0 & 0.0 & 0.0 & 0.0 & 0.0 & 0.0 & 0.0 & 0.0 & 0.0 & 0.0 & 0.0 & 0.0 & 0.0 & 0.0 & 0.0 & 0.0 & 0.0 & 0.0 & 0.0 & 0.0 & 0.0 & 0.0\end{array}$

$\begin{array}{lllllllllllllllllllllllll}0.0 & 0.0 & 0.0 & 0.0 & 0.0 & 0.0 & 0.9 & 0.9 & 3.5 & 0.0 & 1.8 & 1.3 & 1.0 & 0.0 & 0.0 & 0.0 & 0.0 & 0.0 & 1.9 & 0.0 & 1.3 & 0.9 & 1.8 & 0.0 & 0.0\end{array}$

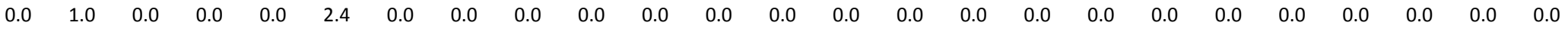

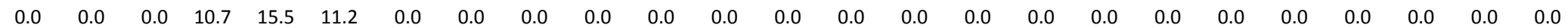

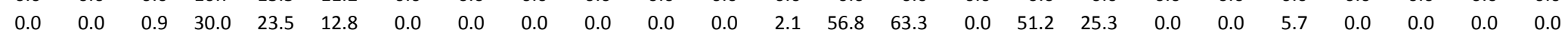

Eunotia praerupto

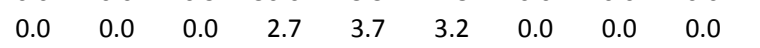

GMC Data Report 404 


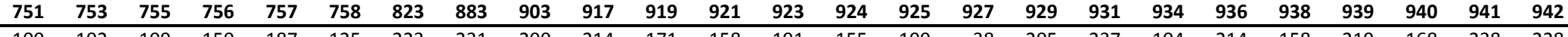

\begin{tabular}{|c|c|c|c|c|c|c|c|c|c|c|c|c|c|c|c|c|c|c|c|c|c|c|c|c|c|}
\hline Sum & 100 & 102 & 109 & 150 & 187 & 125 & 223 & 231 & 200 & 214 & 171 & 158 & 191 & 155 & 199 & 38 & 205 & 237 & 104 & 214 & 158 & 219 & 168 & 238 & 228 \\
\hline Fragilaria leptostauron & 0.0 & 0.0 & 0.0 & 0.0 & 0.0 & 0.0 & 0.4 & 0.0 & 0.0 & 0.0 & 0.0 & 0.0 & 0.0 & 0.0 & 0.0 & 0.0 & 0.0 & 0.0 & 0.0 & 0.0 & 0.0 & 0.0 & 0.0 & 0.0 & 0.0 \\
\hline Frustulia rhomboides & 0.0 & 0.0 & 0.0 & 0.0 & 0.0 & 0.0 & 0.0 & 0.0 & 0.0 & 0.0 & 0.6 & 0.0 & 0.0 & 0.0 & 0.5 & 0.0 & 0.0 & 0.0 & 0.0 & 0.0 & 0.6 & 0.5 & 1.8 & 0.8 & 0.0 \\
\hline Nitzschia gracilis & 0.0 & 1.0 & 0.0 & 0.0 & 0.0 & 1.6 & 0.0 & 0.0 & 0.0 & 0.0 & 0.0 & 0.0 & 0.0 & 0.0 & 0.0 & 0.0 & 0.0 & 0.0 & 0.0 & 0.0 & 0.0 & 0.0 & 0.0 & 0.0 & 0.0 \\
\hline Pinnularia subcapitata & 0.0 & 0.0 & 0.0 & 2.7 & 2.7 & 0.8 & 0.0 & 0.0 & 0.0 & 0.0 & 0.0 & 0.0 & 0.0 & 0.0 & 0.0 & 0.0 & 4.4 & 3.4 & 0.0 & 0.0 & 1.9 & 1.4 & 1.2 & 0.0 & 0.0 \\
\hline Pinnularia subsolaris & 0.0 & 0.0 & 0.0 & 0.0 & 0.0 & 0.0 & 0.0 & 0.0 & 0.0 & 0.0 & 0.0 & 0.0 & 0.0 & 0.0 & 0.0 & 0.0 & 0.0 & 0.0 & 0.0 & 0.0 & 0.0 & 0.0 & 0.0 & 0.0 & 0.0 \\
\hline Tabellaria fenestrata & 0.0 & 0.0 & 0.0 & 0.0 & 0.0 & 0.0 & 0.0 & 0.0 & 0.0 & 0.0 & 0.0 & 0.0 & 0.0 & 0.0 & 0.0 & 0.0 & 0.0 & 0.0 & 0.0 & 0.0 & 0.0 & 0.0 & 0.0 & 0.0 & 0.0 \\
\hline Tabellaria flocculosa & 0.0 & 0.0 & 0.0 & 0.0 & 0.0 & 0.0 & 0.4 & 1.7 & 0.5 & 0.9 & 0.6 & 0.0 & 1.0 & 2.6 & 0.0 & 0.0 & 0.0 & 0.4 & 1.0 & 0.5 & 8.2 & 9.1 & 0.0 & 0.0 & 0.0 \\
\hline Navicula ramosissima & 0.0 & 0.0 & 0.0 & 0.0 & 0.0 & 0.0 & 0.0 & 0.0 & 0.0 & 0.0 & 0.0 & 0.0 & 0.0 & 0.0 & 0.0 & 0.0 & 0.0 & 0.0 & 0.0 & 0.0 & 0.0 & 0.0 & 0.0 & 0.0 & 0.0 \\
\hline Neidium bisulcatum & 0.0 & 0.0 & 0.0 & 0.0 & 0.0 & 0.0 & 0.0 & 0.0 & 0.0 & 0.0 & 0.0 & 0.0 & 0.0 & 0.0 & 0.0 & 0.0 & 4.9 & 6.3 & 0.0 & 0.0 & 0.0 & 0.0 & 0.0 & 0.0 & 0.0 \\
\hline Cyclotella meneghiniana & 0.0 & 0.0 & 1.8 & 0.7 & 0.0 & 0.0 & 0.0 & 0.0 & 0.0 & 0.0 & 0.0 & 0.0 & 0.0 & 0.0 & 0.0 & 0.0 & 2.4 & 0.0 & 5.8 & 3.7 & 10.8 & 9.1 & 3.0 & 2.9 & 0.0 \\
\hline Caloneis westii & 0.0 & 0.0 & 0.0 & 0.0 & 0.0 & 0.0 & 0.0 & 0.0 & 1.5 & 0.0 & 0.0 & 0.0 & 0.0 & 0.0 & 0.0 & 0.0 & 0.0 & 0.0 & 0.0 & 0.0 & 0.0 & 0.0 & 0.0 & 0.0 & 0.0 \\
\hline Eunotia tenella & 0.0 & 0.0 & 0.0 & 5.3 & 5.9 & 7.2 & 0.0 & 0.0 & 0.0 & 0.0 & 0.0 & 0.0 & 0.0 & 0.0 & 0.0 & 0.0 & 0.0 & 0.0 & 0.0 & 0.0 & 0.0 & 0.0 & 0.0 & 0.0 & 0.0 \\
\hline Fragilaria construens var. binodis & 0.0 & 0.0 & 0.0 & 0.0 & 0.0 & 0.0 & 0.0 & 0.0 & 0.0 & 0.0 & 0.0 & 0.0 & 0.0 & 0.0 & 0.0 & 0.0 & 0.0 & 0.0 & 1.0 & 6.1 & 3.2 & 0.0 & 0.0 & 3.8 & 3.9 \\
\hline Navicula capitata var. hungarica & 0.0 & 0.0 & 0.0 & 0.0 & 0.0 & 0.0 & 0.0 & 0.0 & 0.0 & 0.0 & 0.0 & 0.6 & 0.0 & 0.0 & 0.0 & 0.0 & 0.0 & 0.0 & 0.0 & 0.0 & 0.0 & 0.0 & 0.0 & 0.0 & 0.0 \\
\hline Pinnularia major & 0.0 & 0.0 & 0.0 & 0.0 & 1.6 & 0.8 & 0.0 & 0.0 & 0.0 & 0.0 & 0.0 & 0.0 & 0.0 & 0.0 & 0.0 & 0.0 & 0.0 & 0.0 & 0.0 & 0.0 & 0.0 & 0.0 & 0.0 & 0.0 & 0.0 \\
\hline Aulacoseira distans & 1.0 & 0.0 & 0.0 & 11.3 & 7.5 & 16.0 & 0.0 & 0.0 & 0.0 & 0.0 & 0.0 & 0.0 & 0.0 & 0.0 & 0.0 & 0.0 & 0.0 & 0.0 & 0.0 & 0.0 & 0.0 & 0.0 & 0.0 & 0.0 & 0.0 \\
\hline Navicula subtilissima & 0.0 & 0.0 & 0.0 & 0.0 & 0.0 & 0.0 & 0.0 & 0.0 & 0.0 & 0.0 & 0.0 & 0.0 & 0.0 & 0.0 & 0.0 & 0.0 & 0.0 & 0.0 & 0.0 & 0.0 & 0.0 & 0.0 & 0.0 & 0.0 & 0.0 \\
\hline Fragilariforma constricta & 0.0 & 0.0 & 0.0 & 0.0 & 0.0 & 0.0 & 0.0 & 0.0 & 0.0 & 0.0 & 0.0 & 0.0 & 0.0 & 0.0 & 0.0 & 0.0 & 0.0 & 0.0 & 0.0 & 0.0 & 0.0 & 0.0 & 0.0 & 0.0 & 0.0 \\
\hline Achnanthes clevei & 0.0 & 0.0 & 0.0 & 0.0 & 0.0 & 0.0 & 0.0 & 0.0 & 0.0 & 1.9 & 0.0 & 0.0 & 0.0 & 0.0 & 0.0 & 0.0 & 0.0 & 0.0 & 1.9 & 0.0 & 3.2 & 0.0 & 0.0 & 0.0 & 0.0 \\
\hline Cyclotella radiosa & 1.0 & 0.0 & 0.0 & 0.7 & 0.5 & 2.4 & 0.0 & 0.0 & 0.0 & 0.0 & 0.0 & 0.0 & 0.0 & 0.0 & 0.0 & 0.0 & 0.0 & 0.0 & 0.0 & 0.0 & 0.0 & 0.0 & 0.0 & 0.0 & 0.0 \\
\hline Fragilaria construens var. venter & 0.0 & 0.0 & 0.0 & 0.0 & 0.0 & 0.0 & 3.6 & 0.0 & 0.0 & 2.8 & 6.4 & 1.3 & 8.9 & 0.0 & 0.0 & 0.0 & 0.0 & 0.4 & 0.0 & 0.9 & 7.6 & 3.2 & 4.2 & 10.1 & 5.3 \\
\hline Navicula ignota var. palustris & 0.0 & 0.0 & 0.0 & 1.3 & 0.5 & 0.8 & 0.0 & 0.0 & 0.0 & 0.0 & 0.0 & 0.0 & 0.0 & 0.0 & 0.0 & 0.0 & 0.0 & 0.0 & 0.0 & 0.0 & 0.0 & 0.0 & 0.0 & 0.0 & 0.0 \\
\hline
\end{tabular}




$\begin{array}{lllllllllllllllllllll}0.4 & 0.4 & 4.4 & 2.2 & 7.5 & 7.2 & 6.6 & 7.1 & 7.2 & 4.3 & 1.1 & 3.2 & 2.8 & 7.4 & 4.5 & 7.7 & 1.5 & 1.2 & 2.7\end{array}$

Actinoptychus senarius

Caloneis brevis

$\begin{array}{lllllllllllllllllll}0.0 & 0.0 & 0.0 & 0.0 & 0.0 & 0.0 & 0.0 & 0.0 & 0.0 & 0.0 & 0.0 & 0.0 & 0.0 & 0.0 & 0.0 & 0.0 & 0.0 & 0.0 & 0.0\end{array}$

Cocconeis peltoides

$\begin{array}{lllllllllllllllllll}0.4 & 0.4 & 0.0 & 0.0 & 5.8 & 5.9 & 6.6 & 2.4 & 2.2 & 3.3 & 3.8 & 1.1 & 3.5 & 3.4 & 7.1 & 0.5 & 0.0 & 0.0 & 0.7\end{array}$

Delphineis surirella

$\begin{array}{lllllllllllllllllll}5.2 & 8.9 & 11.0 & 19.6 & 24.3 & 25.3 & 33.8 & 29.2 & 18.8 & 26.8 & 15.8 & 12.4 & 15.4 & 25.1 & 24.4 & 36.1 & 14.1 & 5.3 & 19.3\end{array}$

Diploneis smithii

$\begin{array}{rrrrrrrrrrrrrrrrrrr}5.2 & 8.9 & 11.0 & 19.6 & 24.3 & 25.3 & 33.8 & 29.2 & 18.8 & 26.8 & 15.8 & 12.4 & 15.4 & 25.1 & 24.4 & 36.1 & 14.1 & 5.3 & 19.3 \\ 0.0 & 0.0 & 0.0 & 0.0 & 0.4 & 0.0 & 0.4 & 0.0 & 0.6 & 0.0 & 1.1 & 0.0 & 0.7 & 0.0 & 0.6 & 0.0 & 0.0 & 0.0 & 0.0\end{array}$

Gyrosigma wansbeckil

Navicula distans

Navicula palpebralis

Navicula forcipato

Navicula species 1

Nitzschia socialis

Odontella aurita

Paralia sulcata

Pinnularia cruciformis

Plagiogramma vanheurckii

Surirella fastuosa

Thalassionema nitzschioides

Thalassiosira eccentrica

Achnanthes delicatula

Cyclotella striata

Diploneis interrupta

Navicula digitoradiata

Navicula peregrina

Navicula salinarum

Nitzschia obtusa

Nitzschia sigma

Nitzschia vitrea

Rhopalodia operculato

Surirella ovalis

Synedra fasciculata

Amphora veneta

Luticola mutica

Navicula cari var cincta

Nitzschia frustulum

Nitzschia tryblionella

Achnanthes exigua

Achnanthes lanceolata

Amphora ovalis

$\begin{array}{lllllllllllllllllll}0.0 & 0.0 & 0.4 & 0.0 & 0.0 & 0.0 & 0.0 & 1.4 & 1.7 & 1.0 & 0.0 & 1.1 & 0.0 & 0.0 & 0.0 & 0.5 & 0.0 & 0.6 & 0.0 \\ 0.0 & 0.0 & 0.0 & 0.0 & 0.0 & 0.0 & 0.0 & 0.0 & 0.0 & 0.0 & 0.0 & 0.0 & 0.0 & 0.0 & 0.0 & 0.0 & 0.0 & 0.0 & 0.0\end{array}$

$\begin{array}{lllllllllllllllllll}0.0 & 0.0 & 0.0 & 0.0 & 0.0 & 0.0 & 0.0 & 0.0 & 0.6 & 1.0 & 0.0 & 0.0 & 0.0 & 0.0 & 0.0 & 0.0 & 0.0 & 0.0 & 0.0\end{array}$

$\begin{array}{lllllllllllllllllll}0.0 & 0.0 & 0.0 & 0.0 & 0.0 & 0.0 & 0.0 & 0.0 & 0.6 & 1.9 & 0.5 & 1.1 & 0.0 & 0.0 & 0.0 & 1.0 & 2.0 & 2.4 & 0.0\end{array}$

$\begin{array}{lllllllllllllllllll}1.3 & 3.1 & 3.1 & 2.6 & 2.2 & 0.0 & 0.0 & 0.0 & 2.8 & 3.8 & 4.4 & 5.4 & 7.0 & 1.7 & 3.8 & 1.0 & 0.5 & 2.4 & 3.3\end{array}$

$\begin{array}{lllllllllllllllllll}0.0 & 0.0 & 0.0 & 0.0 & 0.0 & 0.0 & 0.0 & 0.0 & 0.0 & 0.0 & 0.0 & 0.0 & 0.0 & 0.0 & 0.0 & 0.0 & 0.0 & 0.0 & 0.0\end{array}$

$\begin{array}{lllllllllllllllllll}0.0 & 0.0 & 0.4 & 0.0 & 0.0 & 0.4 & 0.0 & 0.5 & 0.0 & 0.0 & 2.2 & 0.0 & 0.0 & 0.0 & 0.0 & 0.5 & 0.0 & 0.0 & 0.0\end{array}$

$\begin{array}{rrrrrrrrrrrrrrrrrrr}3.1 & 5.4 & 18.9 & 3.5 & 31.4 & 33.8 & 18.9 & 9.4 & 19.9 & 11.0 & 8.2 & 7.6 & 10.5 & 6.3 & 10.9 & 23.2 & 9.6 & 5.9 & 12.0\end{array}$

$\begin{array}{lllllllllllllllllll}0.0 & 0.0 & 0.0 & 0.0 & 0.0 & 0.0 & 0.0 & 0.0 & 0.0 & 0.0 & 0.0 & 0.0 & 0.0 & 0.0 & 0.0 & 0.0 & 0.0 & 0.0 & 0.0\end{array}$

$\begin{array}{lllllllllllllllllll}0.0 & 0.0 & 0.0 & 0.0 & 0.0 & 0.0 & 0.0 & 0.0 & 0.0 & 0.0 & 0.0 & 0.0 & 0.0 & 0.0 & 0.0 & 0.0 & 0.0 & 0.0 & 0.0\end{array}$

$\begin{array}{lllllllllllllllllll}0.0 & 0.0 & 0.4 & 0.0 & 0.4 & 0.0 & 0.0 & 0.0 & 0.6 & 0.0 & 0.0 & 0.0 & 0.0 & 0.6 & 0.0 & 0.0 & 0.0 & 1.8 & 5.3\end{array}$

$\begin{array}{lllllllllllllllllll}0.0 & 0.4 & 0.4 & 0.0 & 2.2 & 0.0 & 0.4 & 1.4 & 6.1 & 3.8 & 3.8 & 2.7 & 2.8 & 1.1 & 5.8 & 1.5 & 5.6 & 0.0 & 1.3\end{array}$

$\begin{array}{rllllllllllllllllll}0.0 & 0.0 & 0.0 & 3.9 & 4.9 & 7.2 & 3.1 & 5.7 & 11.6 & 13.9 & 4.9 & 3.2 & 7.7 & 16.6 & 7.7 & 11.3 & 12.6 & 1.2 & 2.0\end{array}$

$\begin{array}{lllllllllllllllllll}0.9 & 0.0 & 0.0 & 0.0 & 2.2 & 3.0 & 6.6 & 5.7 & 13.8 & 12.4 & 3.8 & 6.5 & 2.8 & 3.4 & 5.1 & 5.2 & 11.6 & 2.4 & 7.3\end{array}$

$\begin{array}{lllllllllllllllllll}0.0 & 0.0 & 11.0 & 2.6 & 1.8 & 1.7 & 1.3 & 24.1 & 4.4 & 1.9 & 5.5 & 3.2 & 14.7 & 18.9 & 19.2 & 4.1 & 3.0 & 0.6 & 4.0\end{array}$

$\begin{array}{lllllllllllllllllll}0.0 & 0.0 & 0.0 & 0.0 & 0.0 & 0.0 & 0.0 & 0.0 & 0.0 & 0.0 & 0.0 & 0.0 & 0.0 & 0.0 & 0.0 & 0.0 & 0.0 & 0.0 & 0.0\end{array}$

$\begin{array}{lllllllllllllllllll}0.0 & 0.0 & 0.0 & 0.0 & 0.0 & 0.0 & 0.0 & 0.0 & 0.0 & 0.0 & 0.0 & 0.0 & 0.0 & 0.0 & 0.0 & 0.0 & 0.0 & 0.0 & 0.0\end{array}$

$\begin{array}{lllllllllllllllllll}9.6 & 2.2 & 2.6 & 20.0 & 4.9 & 4.2 & 13.2 & 4.7 & 1.7 & 3.3 & 8.2 & 7.6 & 16.1 & 2.3 & 2.6 & 0.5 & 1.5 & 3.6 & 6.0\end{array}$

$\begin{array}{lllllllllllllllllll}0.0 & 0.0 & 0.0 & 0.0 & 0.0 & 0.0 & 0.0 & 0.0 & 0.0 & 0.0 & 0.0 & 0.0 & 0.0 & 0.0 & 0.0 & 0.0 & 0.0 & 0.0 & 0.0\end{array}$

$\begin{array}{lllllllllllllllllll}0.0 & 0.0 & 0.4 & 0.0 & 0.4 & 0.4 & 0.4 & 0.0 & 0.0 & 0.0 & 0.0 & 0.0 & 0.0 & 0.0 & 0.0 & 0.0 & 0.0 & 0.0 & 0.0\end{array}$

$\begin{array}{lllllllllllllllllll}1.7 & 1.3 & 0.9 & 2.6 & 4.4 & 7.6 & 1.8 & 4.7 & 1.1 & 0.0 & 6.0 & 5.4 & 10.5 & 8.6 & 4.5 & 0.0 & 0.5 & 2.4 & 4.7\end{array}$

$\begin{array}{lllllllllllllllllll}0.0 & 0.0 & 0.0 & 0.0 & 0.0 & 0.0 & 0.0 & 0.0 & 0.0 & 0.0 & 0.0 & 0.0 & 0.0 & 0.0 & 0.0 & 0.0 & 0.0 & 0.0 & 0.0\end{array}$

$\begin{array}{lllllllllllllllllll}0.0 & 0.0 & 0.0 & 0.0 & 0.0 & 0.0 & 0.4 & 0.0 & 0.0 & 0.0 & 0.0 & 0.0 & 0.0 & 0.0 & 0.6 & 0.0 & 0.0 & 0.0 & 0.0\end{array}$

$\begin{array}{lllllllllllllllllll}0.0 & 0.0 & 0.0 & 0.0 & 0.0 & 0.0 & 0.0 & 0.0 & 0.0 & 0.0 & 0.0 & 0.0 & 0.0 & 0.0 & 0.0 & 0.0 & 0.0 & 0.0 & 0.0\end{array}$

$\begin{array}{lllllllllllllllllll}9.6 & 2.7 & 0.0 & 0.0 & 0.0 & 0.0 & 0.0 & 1.4 & 0.0 & 0.0 & 2.2 & 0.0 & 0.7 & 0.0 & 0.0 & 0.0 & 0.0 & 3.6 & 0.0\end{array}$

$\begin{array}{lllllllllllllllllll}0.0 & 0.0 & 0.0 & 0.0 & 0.0 & 0.0 & 0.0 & 0.0 & 0.0 & 0.0 & 0.0 & 0.0 & 0.0 & 0.0 & 0.0 & 0.0 & 0.0 & 0.0 & 0.0\end{array}$

$\begin{array}{lllllllllllllllllll}0.0 & 0.0 & 0.0 & 0.0 & 0.0 & 0.0 & 0.0 & 0.0 & 0.0 & 0.0 & 0.0 & 0.0 & 0.0 & 0.0 & 0.0 & 0.0 & 0.0 & 0.0 & 0.0\end{array}$

$\begin{array}{rllllllllllllllllll}0.0 & 0.0 & 0.0 & 0.0 & 0.0 & 0.0 & 0.0 & 0.0 & 0.0 & 0.0 & 0.0 & 1.6 & 0.0 & 0.0 & 0.0 & 0.0 & 2.5 & 10.7 & 2.0\end{array}$

$\begin{array}{lllllllllllllllllll}0.0 & 4.0 & 2.6 & 0.0 & 0.0 & 0.0 & 0.0 & 0.0 & 0.0 & 0.0 & 0.0 & 0.0 & 0.0 & 0.0 & 0.0 & 0.0 & 0.0 & 0.0 & 0.0\end{array}$

$\begin{array}{lllllllllllllllllll}0.0 & 0.0 & 0.0 & 0.0 & 0.0 & 0.0 & 0.0 & 0.0 & 0.0 & 0.0 & 0.0 & 0.0 & 0.0 & 0.0 & 0.0 & 0.0 & 0.0 & 0.0 & 0.0\end{array}$

$\begin{array}{lllllllllllllllllll}0.0 & 0.0 & 0.0 & 0.0 & 0.0 & 0.0 & 0.0 & 0.0 & 0.0 & 0.0 & 0.0 & 0.0 & 0.0 & 0.0 & 0.0 & 0.0 & 0.0 & 0.0 & 0.0\end{array}$

$\begin{array}{lllllllllllllllllll}0.0 & 0.0 & 0.0 & 0.0 & 0.0 & 0.0 & 0.0 & 0.0 & 0.0 & 0.0 & 0.0 & 0.0 & 0.0 & 0.0 & 0.0 & 0.0 & 0.0 & 0.0 & 0.0\end{array}$

$\begin{array}{lllllllllllllllllll}0.0 & 0.4 & 0.4 & 0.4 & 0.4 & 0.0 & 0.4 & 0.5 & 0.0 & 1.0 & 1.1 & 1.1 & 0.0 & 0.6 & 0.0 & 0.0 & 0.5 & 7.1 & 0.7\end{array}$

Caloneis bacillum

Caloneis ventricosa

$\begin{array}{lllllllllllllllllll}0.0 & 0.0 & 0.0 & 0.0 & 0.0 & 0.0 & 0.0 & 0.0 & 0.0 & 0.0 & 0.0 & 0.0 & 0.0 & 0.0 & 0.0 & 0.0 & 0.0 & 3.0 & 3.3\end{array}$

Ceratoneis arcus

$\begin{array}{lllllllllllllllllll}0.0 & 0.0 & 0.0 & 0.0 & 0.0 & 0.0 & 0.0 & 0.0 & 0.0 & 0.0 & 0.0 & 0.0 & 0.0 & 0.0 & 0.0 & 0.0 & 0.0 & 0.0 & 0.0 \\ 0.0 & 0.0 & 0.0 & 0.0 & 0.0 & 0.0 & 0.0 & 0.0 & 0.0 & 0.0 & 0.0 & 0.0 & 0.0 & 0.0 & 0.0 & 0.0 & 0.5 & 0.0 & 0.0\end{array}$




\begin{tabular}{|c|c|c|c|c|c|c|c|c|c|c|c|c|c|c|c|c|c|c|c|}
\hline Sum & 229 & 224 & 227 & 230 & 226 & 237 & 228 & 212 & 181 & 209 & 183 & 185 & 143 & 175 & 156 & 194 & 198 & 169 & 150 \\
\hline Cymbella affinis & 0.0 & 0.0 & 0.0 & 0.0 & 0.0 & 0.0 & 0.0 & 0.0 & 0.0 & $\overline{0.0}$ & 0.0 & 0.0 & 0.0 & 0.0 & 0.0 & 0.0 & 0.0 & 0.0 & $\overline{0.0}$ \\
\hline Cymbella cistula & 0.0 & 0.0 & 0.4 & 0.0 & 0.0 & 0.0 & 0.0 & 0.0 & 0.0 & 0.5 & 0.0 & 0.0 & 0.0 & 0.0 & 0.0 & 0.0 & 0.0 & 0.0 & 0.0 \\
\hline Cymbella ventricosa & 0.0 & 0.0 & 0.0 & 0.0 & 0.0 & 0.0 & 0.0 & 0.0 & 0.0 & 0.0 & 0.0 & 0.0 & 0.0 & 0.0 & 0.0 & 0.0 & 0.0 & 0.0 & 0.0 \\
\hline Diploneis ovalis & 0.4 & 2.2 & 0.0 & 0.0 & 0.4 & 0.0 & 0.0 & 0.0 & 0.0 & 0.0 & 0.0 & 0.0 & 0.0 & 0.0 & 0.0 & 0.0 & 0.0 & 0.0 & 0.0 \\
\hline Epithemia turgida & 0.0 & 0.0 & 0.0 & 0.0 & 0.0 & 0.0 & 0.0 & 0.0 & 0.0 & 0.0 & 0.5 & 0.0 & 0.0 & 0.0 & 0.0 & 0.0 & 0.0 & 0.0 & 0.0 \\
\hline Eunotia germainii & 0.0 & 0.0 & 0.0 & 0.0 & 0.0 & 0.0 & 0.0 & 0.0 & 0.0 & 0.0 & 0.0 & 0.0 & 0.0 & 0.0 & 0.0 & 0.0 & 0.0 & 0.0 & 0.0 \\
\hline Eunotia lunaris & 0.0 & 0.0 & 0.0 & 0.0 & 0.4 & 0.0 & 0.0 & 0.0 & 0.0 & 0.0 & 0.0 & 0.0 & 0.0 & 0.0 & 0.0 & 0.0 & 0.0 & 0.0 & 0.0 \\
\hline Eunotia pectinalis & 0.0 & 0.0 & 0.0 & 0.4 & 0.0 & 0.0 & 0.0 & 0.0 & 0.0 & 0.0 & 0.0 & 0.0 & 0.0 & 0.0 & 0.0 & 0.0 & 0.0 & 0.0 & 0.0 \\
\hline Fragilaria construens & 0.0 & 0.0 & 0.0 & 0.0 & 0.0 & 0.0 & 0.0 & 0.0 & 0.0 & 0.0 & 0.0 & 0.0 & 0.0 & 0.0 & 0.0 & 0.0 & 0.0 & 0.0 & 0.0 \\
\hline Fragilaria vaucheriae & 0.0 & 0.0 & 0.0 & 0.0 & 0.0 & 0.0 & 0.0 & 0.0 & 0.0 & 0.0 & 0.0 & 0.0 & 0.0 & 0.0 & 0.0 & 0.0 & 0.0 & 0.0 & 0.0 \\
\hline Fragilariforma virescens & 0.0 & 0.0 & 0.0 & 0.0 & 0.0 & 0.0 & 0.0 & 0.0 & 0.0 & 0.0 & 0.0 & 0.0 & 0.0 & 0.0 & 0.0 & 0.0 & 0.0 & 0.0 & 0.0 \\
\hline Gomphonema acuminatum & 0.0 & 0.0 & 0.0 & 0.0 & 0.0 & 0.0 & 0.0 & 0.0 & 0.0 & 0.0 & 0.0 & 0.0 & 0.0 & 0.0 & 0.0 & 0.0 & 0.0 & 0.0 & 0.0 \\
\hline Gomphonema gracile & 0.0 & 0.0 & 0.0 & 0.0 & 0.0 & 0.0 & 0.0 & 0.0 & 0.0 & 0.0 & 0.0 & 0.0 & 0.0 & 0.0 & 0.0 & 0.0 & 0.0 & 0.0 & 0.0 \\
\hline Meridion circulare & 0.0 & 0.0 & 0.0 & 0.0 & 0.0 & 0.0 & 0.0 & 0.0 & 0.0 & 0.0 & 0.0 & 0.0 & 0.0 & 0.0 & 0.0 & 0.0 & 0.0 & 0.0 & 0.0 \\
\hline Navicula begeri & 0.0 & 0.0 & 0.0 & 0.0 & 0.0 & 0.0 & 0.0 & 0.0 & 0.0 & 0.0 & 0.0 & 0.0 & 0.0 & 0.0 & 0.0 & 0.0 & 0.0 & 0.0 & 0.0 \\
\hline Navicula brockmanii & 10.5 & 4.5 & 4.8 & 0.0 & 0.0 & 0.0 & 0.4 & 0.5 & 0.6 & 3.8 & 9.3 & 10.8 & 0.0 & 0.0 & 0.0 & 0.5 & 10.1 & 7.7 & 0.0 \\
\hline Navicula pupula & 0.0 & 0.0 & 0.0 & 0.0 & 0.0 & 0.0 & 0.0 & 0.0 & 0.0 & 0.5 & 1.6 & 3.2 & 0.0 & 0.0 & 0.0 & 0.0 & 1.0 & 5.3 & 0.0 \\
\hline Navicula pusilla & 1.3 & 2.2 & 0.4 & 0.9 & 0.4 & 0.0 & 0.0 & 0.0 & 0.0 & 0.5 & 2.2 & 3.2 & 0.0 & 0.0 & 0.0 & 0.5 & 1.0 & 1.2 & 3.3 \\
\hline Navicula radiosa & 0.0 & 0.0 & 0.0 & 0.0 & 0.0 & 0.0 & 0.0 & 0.0 & 0.0 & 0.0 & 0.0 & 0.0 & 0.0 & 0.0 & 0.0 & 0.0 & 0.0 & 0.0 & 0.0 \\
\hline Navicula tripunctata & 0.0 & 0.0 & 0.0 & 0.0 & 0.0 & 0.0 & 0.0 & 0.0 & 0.0 & 0.0 & 0.0 & 0.0 & 0.0 & 0.0 & 0.0 & 0.0 & 0.0 & 0.0 & 0.0 \\
\hline Navicula viridula & 0.9 & 1.8 & 0.9 & 0.0 & 0.0 & 0.0 & 0.0 & 0.0 & 0.0 & 0.0 & 0.0 & 0.0 & 0.0 & 0.0 & 0.0 & 0.0 & 0.0 & 0.0 & 0.0 \\
\hline Nitzschia fruticosa & 7.4 & 8.9 & 3.5 & 0.0 & 0.0 & 0.0 & 0.0 & 0.0 & 0.0 & 0.0 & 0.0 & 0.0 & 0.0 & 0.0 & 0.0 & 0.0 & 0.0 & 0.0 & 0.0 \\
\hline Nitzschia linearis & 1.3 & 1.3 & 3.1 & 1.3 & 0.0 & 0.0 & 2.2 & 0.0 & 0.0 & 0.0 & 0.0 & 0.0 & 0.0 & 0.0 & 0.0 & 0.0 & 0.0 & 0.0 & 0.0 \\
\hline Nitzschia palea & 4.4 & 13.4 & 4.4 & 9.6 & 0.9 & 0.8 & 0.0 & 0.0 & 0.0 & 0.0 & 1.1 & 5.4 & 0.0 & 0.0 & 0.0 & 0.0 & 1.0 & 2.4 & 7.3 \\
\hline Nitzschia palustris & 0.4 & 0.9 & 0.0 & 0.4 & 0.0 & 0.0 & 0.0 & 0.0 & 0.0 & 0.5 & 0.0 & 0.0 & 0.0 & 0.0 & 0.0 & 0.0 & 0.5 & 0.6 & 0.0 \\
\hline Pinnularia abaujensis & 0.0 & 0.0 & 0.0 & 0.0 & 0.0 & 0.0 & 0.0 & 0.0 & 0.0 & 0.0 & 0.0 & 0.0 & 0.0 & 0.0 & 0.0 & 0.0 & 0.0 & 0.0 & 0.0 \\
\hline Pinnularia borealis & 10.0 & 10.3 & 9.7 & 7.0 & 0.9 & 0.0 & 0.0 & 0.0 & 1.1 & 0.5 & 7.7 & 3.2 & 2.1 & 1.7 & 1.3 & 0.0 & 0.0 & 0.0 & 2.0 \\
\hline Pinnularia brevicostata & 4.4 & 0.0 & 0.0 & 6.5 & 0.0 & 0.0 & 0.0 & 0.0 & 0.0 & 0.0 & 0.0 & 0.0 & 0.0 & 0.0 & 0.0 & 0.0 & 0.0 & 0.0 & 0.0 \\
\hline Pinnularia intermedia & 0.0 & 0.0 & 0.0 & 0.0 & 0.0 & 0.0 & 0.0 & 0.0 & 0.0 & 0.0 & 0.0 & 0.0 & 0.0 & 0.0 & 0.0 & 0.0 & 0.0 & 0.0 & 0.0 \\
\hline Pinnularia lagerstedtii & 3.1 & 8.9 & 10.1 & 0.0 & 0.0 & 0.0 & 0.0 & 0.0 & 0.0 & 0.0 & 0.0 & 1.6 & 0.0 & 0.0 & 0.0 & 0.0 & 0.5 & 2.4 & 0.7 \\
\hline Pinnularia mesolepta & 0.0 & 0.0 & 0.0 & 0.0 & 0.0 & 0.0 & 0.0 & 0.0 & 0.0 & 0.0 & 0.0 & 0.0 & 0.0 & 0.0 & 0.0 & 0.0 & 0.0 & 1.8 & 0.0 \\
\hline Pinnularia microstauron & 3.1 & 4.5 & 0.0 & 0.0 & 0.0 & 0.0 & 0.0 & 0.0 & 0.0 & 1.4 & 0.5 & 3.2 & 0.0 & 0.0 & 0.0 & 0.5 & 1.5 & 10.1 & 8.7 \\
\hline Pinnularia viridis & 0.9 & 2.7 & 2.6 & 0.0 & 0.0 & 0.4 & 0.0 & 0.0 & 0.0 & 0.5 & 0.0 & 0.5 & 0.7 & 0.0 & 0.0 & 0.0 & 2.5 & 8.3 & 0.0 \\
\hline Pseudostaurosira brevistriata & 0.0 & 0.0 & 0.0 & 0.0 & 0.0 & 0.0 & 0.0 & 0.0 & 0.0 & 0.0 & 0.0 & 0.0 & 0.0 & 0.0 & 0.0 & 0.0 & 0.0 & 0.0 & 0.0 \\
\hline Stauroneis anceps & 0.0 & 0.0 & 0.0 & 0.0 & 0.0 & 0.0 & 0.0 & 0.0 & 0.0 & 0.0 & 0.0 & 0.0 & 0.0 & 0.0 & 0.0 & 0.0 & 0.0 & 3.6 & 0.0 \\
\hline Stauroneis phoenicentron & 0.0 & 0.0 & 0.0 & 0.0 & 0.0 & 0.4 & 0.0 & 0.0 & 0.0 & 0.0 & 0.0 & 0.0 & 0.0 & 0.0 & 0.0 & 0.0 & 0.0 & 0.0 & 0.0 \\
\hline Staurosirella pinnata & 0.4 & 1.8 & 1.3 & 13.0 & 1.8 & 0.0 & 0.9 & 1.4 & 0.0 & 0.0 & 2.7 & 0.0 & 0.7 & 1.7 & 0.0 & 0.5 & 0.0 & 0.0 & 0.0 \\
\hline Synedra ulna & 0.0 & 0.0 & 0.0 & 0.0 & 0.0 & 0.0 & 0.0 & 0.0 & 0.6 & 0.5 & 0.0 & 0.0 & 0.0 & 0.0 & 0.0 & 0.0 & 0.0 & 0.0 & 0.0 \\
\hline Achnanthes conspicua & 0.0 & 0.0 & 0.0 & 0.0 & 0.0 & 0.0 & 0.0 & 0.0 & 0.0 & 0.0 & 0.0 & 0.0 & 0.0 & 0.0 & 0.0 & 0.0 & 0.0 & 0.0 & 0.0 \\
\hline Eunotia exigua & 0.0 & 0.0 & 0.0 & 0.0 & 0.4 & 0.0 & 0.0 & 0.0 & 0.0 & 0.0 & 0.0 & 0.0 & 0.0 & 0.0 & 0.0 & 0.0 & 0.0 & 0.0 & 0.0 \\
\hline Eunotia praerupta & 0.0 & 0.0 & 0.0 & 0.0 & 0.0 & 0.0 & 0.0 & 0.0 & 0.0 & 0.0 & 0.0 & 0.0 & 0.0 & 0.0 & 0.0 & 0.0 & 0.0 & 0.0 & 0.0 \\
\hline
\end{tabular}




\begin{tabular}{|c|c|c|c|c|c|c|c|c|c|c|c|c|c|c|c|c|c|c|c|}
\hline Sum & 229 & 224 & 227 & 230 & 226 & 237 & 228 & 212 & 181 & 209 & 183 & 185 & 143 & 175 & 156 & 194 & 198 & 169 & 150 \\
\hline Fragilaria leptostauron & 0.4 & 0.0 & 0.0 & 0.0 & 0.0 & 0.0 & 0.0 & 0.0 & 0.0 & 0.0 & 0.0 & 0.0 & 0.7 & 0.0 & 0.6 & 0.0 & 0.5 & 0.0 & 0.0 \\
\hline Frustulia rhomboides & 0.0 & 0.0 & 0.0 & 0.0 & 0.0 & 0.0 & 0.0 & 0.0 & 0.0 & 0.0 & 1.6 & 0.0 & 0.0 & 0.0 & 0.0 & 0.5 & 2.0 & 0.0 & 0.0 \\
\hline Nitzschia gracilis & 0.0 & 0.0 & 0.0 & 0.0 & 0.0 & 0.0 & 0.0 & 0.0 & 0.0 & 0.0 & 0.0 & 0.0 & 0.0 & 0.0 & 0.0 & 0.0 & 0.0 & 0.0 & 0.0 \\
\hline Pinnularia subcapitata & 0.0 & 1.3 & 0.4 & 0.0 & 0.0 & 0.0 & 0.0 & 0.0 & 0.0 & 0.0 & 0.0 & 0.0 & 0.0 & 0.0 & 0.0 & 0.0 & 0.0 & 0.0 & 0.0 \\
\hline Pinnularia subsolaris & 0.0 & 0.0 & 0.0 & 0.0 & 0.0 & 0.0 & 0.0 & 0.0 & 0.0 & 0.0 & 0.0 & 0.0 & 0.0 & 0.0 & 0.0 & 0.0 & 0.0 & 0.0 & 0.0 \\
\hline Tabellaria fenestrata & 0.0 & 0.0 & 0.0 & 0.0 & 0.0 & 0.0 & 0.0 & 0.0 & 0.0 & 0.0 & 0.0 & 0.0 & 0.0 & 0.0 & 0.0 & 0.0 & 0.0 & 0.0 & 0.0 \\
\hline Tabellaria flocculosa & 0.0 & 0.0 & 0.0 & 0.0 & 0.0 & 0.0 & 0.4 & 0.0 & 0.6 & 0.5 & 0.0 & 0.5 & 0.0 & 0.0 & 0.0 & 0.0 & 0.5 & 0.0 & 0.0 \\
\hline Navicula ramosissima & 0.0 & 0.0 & 0.0 & 0.0 & 0.0 & 0.0 & 0.0 & 0.0 & 0.0 & 0.0 & 0.0 & 0.0 & 0.0 & 0.0 & 0.0 & 0.0 & 0.0 & 0.0 & 0.0 \\
\hline Neidium bisulcatum & 0.0 & 0.0 & 0.0 & 0.0 & 0.0 & 0.0 & 0.0 & 0.0 & 0.0 & 0.0 & 0.0 & 0.0 & 0.0 & 0.0 & 0.0 & 0.0 & 0.0 & 0.0 & 0.0 \\
\hline Cyclotella meneghiniana & 8.3 & 4.0 & 0.0 & 0.0 & 0.0 & 0.0 & 0.0 & 0.0 & 3.9 & 1.4 & 0.0 & 4.3 & 0.0 & 0.0 & 0.0 & 1.5 & 12.1 & 3.0 & 2.0 \\
\hline Caloneis westii & 0.0 & 0.0 & 0.0 & 0.0 & 0.0 & 0.0 & 0.4 & 0.0 & 0.0 & 0.0 & 0.0 & 0.5 & 0.7 & 0.6 & 1.3 & 2.1 & 0.5 & 0.0 & 0.7 \\
\hline Eunotia tenella & 0.0 & 0.0 & 0.0 & 0.0 & 0.0 & 0.0 & 0.0 & 0.0 & 0.0 & 0.0 & 0.0 & 0.0 & 0.0 & 0.0 & 0.0 & 0.0 & 0.0 & 0.0 & 0.0 \\
\hline Fragilaria construens var. binodis & 7.0 & 0.4 & 0.0 & 0.0 & 0.0 & 0.0 & 0.0 & 0.0 & 0.0 & 0.0 & 0.0 & 0.0 & 0.0 & 0.0 & 0.0 & 0.0 & 0.0 & 0.0 & 0.0 \\
\hline Navicula capitata var. hungarica & 0.0 & 0.0 & 0.0 & 0.0 & 0.0 & 0.0 & 0.0 & 0.0 & 0.0 & 0.0 & 0.0 & 0.0 & 0.0 & 0.0 & 0.0 & 0.5 & 0.0 & 0.0 & 0.7 \\
\hline Pinnularia major & 0.0 & 0.0 & 0.0 & 0.0 & 0.0 & 0.0 & 0.0 & 0.0 & 0.0 & 0.0 & 0.0 & 0.0 & 0.0 & 0.0 & 0.0 & 0.0 & 0.0 & 0.0 & 0.0 \\
\hline Aulacoseira distans & 0.0 & 0.0 & 0.0 & 0.0 & 0.0 & 0.0 & 0.0 & 0.0 & 0.0 & 0.0 & 0.0 & 0.0 & 0.0 & 0.0 & 0.0 & 0.0 & 0.0 & 0.0 & 0.0 \\
\hline Navicula subtilissima & 0.0 & 0.0 & 0.0 & 0.0 & 0.0 & 0.0 & 0.0 & 0.0 & 0.0 & 0.0 & 0.0 & 0.0 & 0.0 & 0.0 & 0.0 & 0.0 & 0.0 & 0.0 & 0.0 \\
\hline Fragilariforma constricta & 0.0 & 0.0 & 0.0 & 0.0 & 0.0 & 0.0 & 0.0 & 0.0 & 0.0 & 0.0 & 0.0 & 0.0 & 0.0 & 0.0 & 0.0 & 0.0 & 0.0 & 0.0 & 0.0 \\
\hline Achnanthes clevei & 0.9 & 0.0 & 0.0 & 0.0 & 0.0 & 0.0 & 0.0 & 0.0 & 0.0 & 0.0 & 0.0 & 0.0 & 0.0 & 0.0 & 0.0 & 0.0 & 0.0 & 0.0 & 0.0 \\
\hline Cyclotella radiosa & 0.0 & 0.0 & 0.0 & 0.0 & 0.0 & 0.0 & 0.0 & 0.0 & 0.0 & 0.0 & 0.0 & 0.0 & 0.0 & 0.0 & 0.0 & 0.0 & 0.0 & 0.0 & 0.0 \\
\hline Fragilaria construens var. venter & 2.6 & 1.3 & 0.9 & 3.5 & 1.3 & 1.7 & 1.8 & 0.0 & 0.0 & 0.0 & 0.0 & 0.0 & 0.0 & 0.0 & 0.0 & 0.0 & 0.0 & 0.0 & 0.0 \\
\hline Navicula ignota var. palustris & 0.0 & 0.0 & 0.0 & 0.0 & 0.0 & 0.0 & 0.0 & 0.0 & 0.0 & 0.0 & 0.0 & 0.0 & 0.0 & 0.0 & 0.0 & 0.0 & 0.0 & 0.0 & 0.0 \\
\hline
\end{tabular}

